\title{
Uncertainty modeling in reliability analysis of floating wind turbine support structures
}

\author{
Salem Okpokparoro ${ }^{\mathrm{a}, \mathrm{b}}$, Srinivas Sriramula ${ }^{\mathrm{a}, *}$ \\ ${ }^{a}$ University of Aberdeen, Aberdeen, AB24 3UE, United Kingdom \\ ${ }^{b}$ Petroleum Technology Development Fund (PTDF), 2 Memorial Close, Central Business \\ District, Abuja. Nigeria
}

\begin{abstract}
Accurate structural reliability assessment of floating wind turbine (FWT) systems is a desideratum for achieving consistent optimal reliability levels and cost-effective design. Such reliability assessment should consider relevant system uncertainties - a nontrivial task. Formulation of the reliability problem requires structural demand in form of load and load effect. Support structure loads are predicted with time-domain dynamic simulations. This represents a challenge when thousands of such simulations are required to capture the uncertainty associated with design variables. Finite element analysis (FEA) is commonly used to evaluate load effects such as stresses, strains etc. This can be computationally expensive if not prohibitive when such evaluation is carried out for every time step. To tackle these issues, a framework for expeditious load effect computation and robust reliability analysis of FWT support structures under ultimate limit state design is presented. The framework employs linear elastic FEA and Kriging surrogate models. The adequacy of Kriging as applied in this study is investigated using high fidelity simulation data. The results highlight the importance of incorporating the Kriging uncertainty in the formulation of the limit state function. With the framework presented, FWT support structures can be designed at consistent reliability levels leading to cost reductions.
\end{abstract}

Keywords: Uncertainty modeling, Structural reliability, Finite element analysis, Offshore wind turbines, Kriging surrogate model, Floating support structure

\footnotetext{
${ }^{*}$ Corresponding author

Email address: s.sriramula@abdn.ac.uk (Srinivas Sriramula)
} 


\section{Introduction}

Since the industrial revolution, there has been an increasing demand for energy. This demand has mostly been met by energy from fossil fuels such as coal, oil and gas [1]. Only in recent decades that the clamour for green energy necessitated by the drive to tackle climate change has led to a steady growth of renewable energy capacity and output. Similarly, the interest in offshore wind as viable option for tapping into the rich wind resource available offshore has grown significantly in the past decade. Most of the current offshore wind turbine projects have fixed structures for their support. Monopile, jacket and tripod structures are the most widely used types of fixed support structures. These fixed structures are depth limited (usually $<50 m$ water depth) [2]. As the availability of shallow water sites rapidly declines, the need for floating systems better suited for deep water becomes inevitable. There exists a largely untapped market in deep water that is potentially a game changer. However, with floating concepts come additional costs mostly linked to the floating support structure [3]. Tapping into this market would therefor require robust research and development of cost effective floating systems that would withstand environmental loads and accumulated damage throughout the service life of the turbine. The current framework contained in the widely used wind turbine design standards; IEC 61400-1 [4] and IEC 61400-3 [5], can at best be described as semi-probabilistic. The semi-probabilistic design approach uses partial safety factors to account for uncertainties in the design. These partial safety factors most often lead to over-design with adverse cost implication. A probabilistic approach on the other hand explicitly accounts for uncertainties [6], leading to cost effective designs and more rational safety factors. The impediment herein is that such a design would require numerous evaluations of failure condition in the form of a limit state function (LSF). This implies prohibitive computational effort in assessing structural demands given dynamic analysis of these structures is performed in the time domain using aero-hydro-servo-elastic tools. To facilitate quick assessment of computationally-expensive-to-evaluate structural responses, surrogate models have gained popularity. Authors such as [7-9] to mention a few, have carried out reliability-based analysis of wind turbines by employing surrogate models. Morató et al. [7] carried out reliability analysis of a monopile supported wind turbine using Kriging surrogate models. In their study, en- 
vironmental and loading uncertainty was accounted for. The evaluation of the Kriging model uncertainty was not covered neither were key modeling uncertainties included. In the reliability analysis performed in Ref. [8], only environmental uncertainties and the associated metamodel uncertainty was covered. Surrogate modeling in form of Gaussian process regression was also employed by Stieng and Muskulus [9] in the reliability-based design optimization of an offshore wind turbine (OWT) support structure. Yang et al. [10] demonstrated the use Kriging model for reliability-based optimization of a tripod sub-structure. Modeling uncertainties were neglected in their study as well. A substantial amount of the literature on OWT reliability-based analysis have focused on fixed foundation concepts [7-11]. The structural dynamics of FWTs are different from fixed concepts as floating systems are more compliant to environmental loads implying greater variability in structural loading. An approach that accounts for environmental, material, geometric and modeling uncertainties for FWT concepts is still lacking and represents a gap in knowledge this paper attempts to fill.

In this study, Eurocode 1990 [12] is used to quantify the Kriging model uncertainty. The influence of including the Kriging uncertainty in the LSF on computed failure probability is investigated using 1000 Latin Hypercube Samples (LHS). This represents 6000 time-domain simulations given 6 realizations of wind and waves are used. Haid et al. [13] showed that when 5-10 seeds (for 10-min long simulations) are used in ultimate load analysis of the OC3-Hywind spar, the average of the maximum most load channels converges to about $1 \%$ difference from the mean maximum when 36 seeds are used. The mooring tension was not among the structural responses investigated by Haid et al. [13]. The ultimate limit state (ULS) design of mooring lines is usually based on the 50-year line tension [14]. In the present paper, these were calculated using $6 \times 1$-hr long simulations. Other relevant uncertainties are adopted from available literature $[10,15,16]$. To determine ULS design-drivers that produce the most extreme loads, load analysis was performed for the benchmark FWT- the 5MW wind turbine [17] mounted on the OC3-Hywind spar buoy [18]. Three design load cases (DLCs) from IEC 61400-3 [5] were analysed. DLC1.3 and 1.6a from the power production load cases and DLC6.1a from the parked/idling load cases. These DLCs are recommended by IEC 61400-3 [5] for support structure design and do not require load extrapolation. Authors such as $[7,10,15]$ to mention a few, employed similar load cases for ULS reliability-based support structure design. Additionally, in Ref. [19, 20], DLC1.3 was identified as the ULS design 
driver for the OC3-Hywind FWT when the turbine is working under normal conditions. The aero-hydro-servo-elastic tool OpenFAST (formerly known as FAST [21]) developed at the National Renewable Energy Laboratories (NREL) is used for dynamic analysis. Support structure output channels from FAST are mainly sectional forces and moments as well as rotations and translations outputted as time series. For robust structural design, these loads are used to calculate stresses which in turn are used to compute failure criterion. The violation of such failure criterion is the basis for formulating LSFs used in optimization routines and reliability assessment. The evaluation of the LSF becomes computationally prohibitive if such evaluation is to be carried out for each simulation time step, the required number of DLCs and for different wind/wave seeds. Various approaches have been adopted by researchers to avoid such computationally expensive exercise. Young et al. [22] evaluated stress utilization in the optimization of a composite tower for a floating wind turbine (FWT) using the extreme loads from extreme event table generated from aero-hydro-servo-elastic simulations. This is however a conservative approach as in reality the extreme values of the loads are usually not contemporaneous. It is also worth mentioning the work by Muskulus [23] where the use of Pareto-optimal loads was proposed as a potential solution to this issue. Generally speaking, two approaches are common if the computational cost of running finite element (FE) stress analysis for each time step is to be avoided: (1) the combination of univariate maxima which can be highly conservative or (2) the use of contemporaneous loads at a single time step which can lead to underestimation of the design stress as the ultimate stress might not result from the the combination of loads at the chosen time step. Some studies avoided the use of time-domain simulations outright, thereby neglecting the influence of the nonlinear behaviour of the couple wind turbine system [10, 24-26]. To address this issue, a methodology for expeditious evaluation of load effect of FWT support structure from time series output of aero-hydro-servo-elastic simulation is presented. The method leverages on the linearization of FE solution under linear elastic loading. The sensitivity of design loads to environmental, material and geometric uncertainties was also investigated. Velarde et al. [27] performed similar sensitivity analysis but for fatigue loads on an OWT installed on gravity based foundation. Finally we present reliability analysis, employing trained Kriging models and incorporating relevant uncertainties. Given the huge computational requirement of our study, high performance computing infrastructure of the University of Aberdeen (named Maxwell) was used. This provided 200 job slots. 


\section{Framework for reliability analysis}

To achieve a robust design, it is pertinent to evaluate the structural integrity of the components of the FWT support structure. This involves the computation of failure probability of structural components exposed to loading uncertainties. This uncertainty in loading emanates from the randomness of environmental conditions and non-linearities of the coupled wind turbine system. It is also crucial to include material and geometric uncertainties as well as physical and epistemic uncertainties. Epistemic uncertainties such as statistical, simulation and model uncertainties reflect the paucity of knowledge of the environment or system [16]. The reliability framework proposed in this paper accounts for the possible extreme realizations of uncertain parameters the structure would encounter during its service life. Fig. 1 shows the schematic of the proposed framework. As shown in Fig. 1, load analysis is first performed to determine the set of turbine parameters that produce severest loading for each DLC. This set of parameters $(\boldsymbol{\Theta})$ include wind speed, sea state and wind/wave misalignment. For this work, we only consider uncertainties related to wind speed and sea state. Material and geometrical uncertainties denoted by $(\boldsymbol{\Omega})$ result in uncertainties in stiffness and by extension contribute to uncertainty in structural responses. The thickness, density and Young's modulus of the tower are treated as random variables in order to capture this uncertainty. From the distributions of the uncertain parameters described above (making up $n=1,2, \ldots, m$ random variables), LHS is used to generate an experimental design comprising $i=1,2, . . k$ sample points. Aero-hydro-servo-elastic simulations is then carried out for each sample point, and where needed FE stress computation is performed to give the design load effect $\boldsymbol{Y}_{\boldsymbol{i}}^{\boldsymbol{s i m}}$. Due to the computational cost of estimating $\boldsymbol{Y}_{i}^{\boldsymbol{s i m}}$, Kriging surrogate model is trained using the sample points and their corresponding responses. This allows for the load effect to be explicitly defined in terms of the primary input variables $(\boldsymbol{\Theta}, \boldsymbol{\Omega})$ with easy evaluation. Finally after the calibration and validation of the Kriging model, we develop and evaluate LSFs to obtain failure probabilities that account for associated system uncertainties $\boldsymbol{X}$.

\section{Dynamic modeling and DLC simulation}

\subsection{Description of floating wind turbine model}

For conciseness, only a brief description is given of the benchmark FWT; the 5MW wind turbine [17] mounted on the OC3-Hywind spar buoy [18]. The 


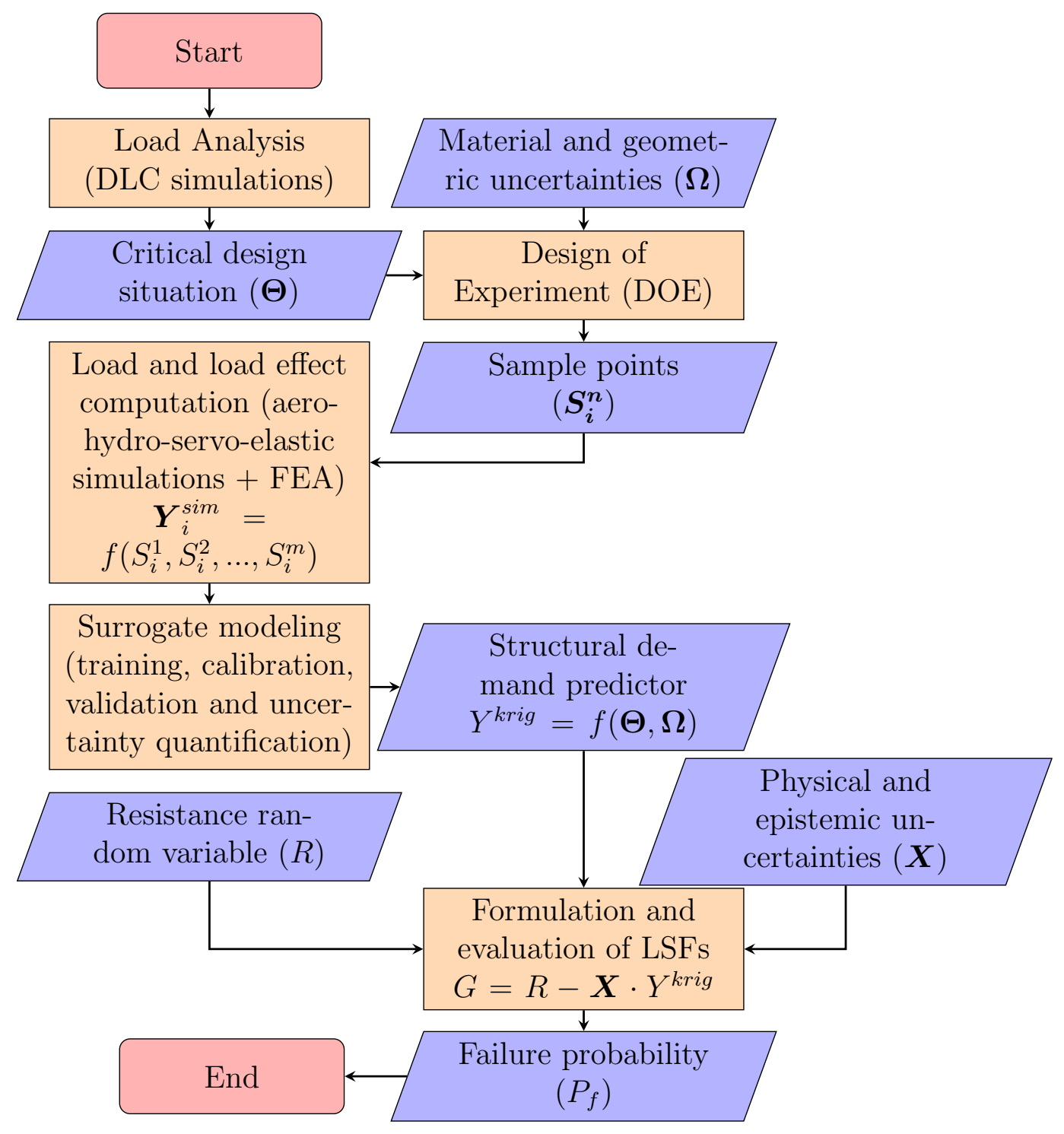

Fig. 1. Schematic representation of reliability framework

spar buoy is a slender draft hull, with ballast in the lower part for stability. For station-keeping, catenary mooring system is adopted. Three catenary mooring lines are connected to the platform through a delta connection (the delta connection increases the yaw stiffness of the mooring) with an angle of $120^{\circ}$ between adjacent lines [18]. The mooring attachment at the fairleads is located at a radius of $5.2 \mathrm{~m}$ from the OC3 platform centreline and a depth of 
$70 m$ below still water level (SWL). A summary of the structural properties of the tower is given in Table 1.

Table 1. Structural properties of OC3-Hywind tower

\begin{tabular}{ll}
\hline Tower base elevation above SWL & $10 \mathrm{~m}$ \\
Tower top elevation above SWL & $87.6 \mathrm{~m}$ \\
Integrated tower mass & $249718 \mathrm{~kg}$ \\
Tower base diameter | thickness & $6.5 \mathrm{~m} \mid 0.027 \mathrm{~m}$ \\
Tower top diameter | thickness & $3.87 \mathrm{~m} \mid 0.019 \mathrm{~m}$ \\
Tower effective density & $8500 \mathrm{~kg} / \mathrm{m}^{3}$ \\
Tower shear modulus | Young's modulus & $80.8 \mathrm{GPa} \mid 210 \mathrm{GPa}$ \\
\hline
\end{tabular}

The OC3 platform is designed for water depths ranging from $200 \mathrm{~m}$ to $700 \mathrm{~m}$. For the sake of generic analysis, $320 \mathrm{~m}$ is the assumed water depth for this work. An illustration of the OC3-Hywind is shown on the right of Fig. 2.

\subsection{Environmental conditions}

According to IEC 61400-3 [5], offshore wind turbine support structures are to be designed based on site-specific environmental conditions. For this study, the Statfjord site located in the Norwegian sector of the northern North sea is chosen as a representative site for the deployment of the FWT. The location of the site can be seen on the left of Fig. 2. The coordinates are $61^{\circ} 15^{\prime} 20^{\prime \prime} \mathrm{N}$ and $1^{\circ} 51^{\prime} 14^{\prime \prime} \mathrm{E}$. Although the water depth at this site is around $150 \mathrm{~m}$, a water depth of $320 \mathrm{~m}$ is assumed for the sake of a generic analysis. To account for the correlation between wind and waves during normal metocean conditions, the joint probabilistic model established by Johannessen et al. [28] for sites in the northern North sea is adopted. Johannessen et al. [28] established conditional distributions of wave height and peak period based on 1-hour averaged wind speed measurements covering the period 1973-1999. Water current data was not available, hence we assume a near-surface current profile with current velocities at SWL of $0.6 \mathrm{~m} / \mathrm{s}$ and $1.2 \mathrm{~m} / \mathrm{s}$ for normal and extreme current loads respectively. The 50-year wind speed at the hub height is taken as $41 \mathrm{~m} / \mathrm{s}$ while the 50 -year wave height $\left(H_{s}\right)$ and peak period $\left(T_{p}\right)$ are assumed to be $8.52 \mathrm{~m}$ and $12.45 \mathrm{~s}$ respectively. 

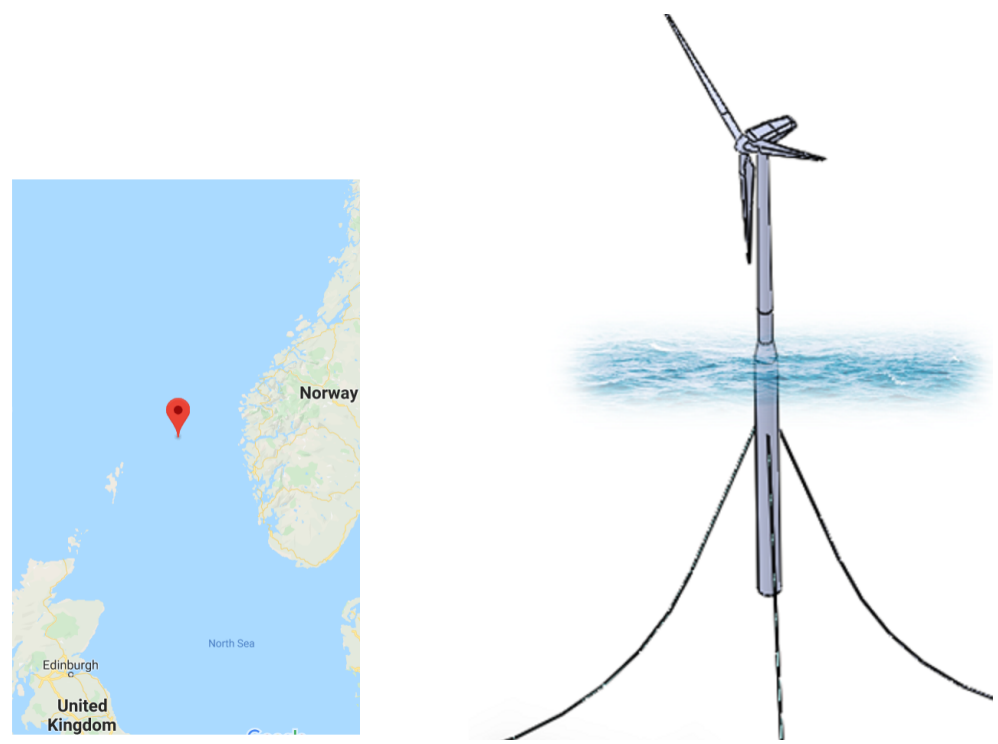

Fig. 2. Location of reference site (Courtesy: www.maps.google.com) [left]; Illustration of OC3-Hywind Spar FWT [Right]

\subsection{Design load case}

The design of an OWT is mostly based on a structural dynamics model that is robust enough to predict the design loads for all relevant combinations of external conditions and design situations, covering the most significant and probable conditions that an OWT may experience. This gives rise to an extensive list of DLCs for which simulating every possible scenario is computationally intensive. For this study, three DLCs from the ULS load set of IEC 61400-3 [5] are selected. Under the power production load cases, DLC1.3 and DLC1.6a are chosen. From parked/idling load cases, DLC6.1a is chosen. For DLC1.3, the wind regime is characterized by the Extreme Turbulence Model (ETM). An irregular Normal Sea State (NSS) model is used with wave height $H_{s}$ conditioned on the mean wind speed $U_{w}$ (measured $10 m$ above SWL). This accounts for the correlation between wind and waves during normal wind conditions. The conditional distribution of $H_{s}$ for a given $U_{w}$ presented by Johannessen et al. [28] is adopted. The expected value of $H_{s}$ is obtained from Equation 1 and the peak period $T_{p}$ is determined with Equation 2.

$$
\begin{gathered}
H_{s}=\beta \Gamma\left(\frac{1}{A}+1\right) \\
T_{p}=\left(4.883+2.68 H_{s}^{0.529}\right)\left[1-0.19\left(\frac{U_{w}-\left(1.764+3.426 H_{s}^{0.78}\right)}{1.764+3.426 H_{s}^{0.78}}\right)\right]
\end{gathered}
$$


where the shape and scale parameters are given by $A=2+0.135 U_{w}$ and $\beta=1.8+0.1 U_{w}^{1.322}$ respectively. Since hub height wind measurements are commonly used in wind turbine analysis, the variation of wind speed with height is estimated with the power law profile given in Equation 3.

$$
U(Z)=U_{\text {ref }}\left(\frac{Z}{Z_{\text {Ref }}}\right)^{\alpha}
$$

where $Z$ is height above SWL, $U(Z)$ is the wind speed at height $Z$, Zref is the reference height above SWL at which wind measurement $U_{\text {ref }}$ is taken, and $\alpha$ is the wind shear or power law exponent. The mean wind speed $U_{w}$ can be computed for any given hub height wind speed using Equation 3 and vice versa.

DLC1.3 requires simulations for the range of wind speeds within the cut-in $\left(U_{\text {in }}=3 \mathrm{~m} / \mathrm{s}\right)$ and cut-out wind speed $\left(U_{\text {out }}=25 \mathrm{~m} / \mathrm{s}\right)$ range of the turbine i.e. $4 \mathrm{~m} / \mathrm{s}-24 \mathrm{~m} / \mathrm{s}$. In this section, a bin interval of $2 \mathrm{~m} / \mathrm{s}$ is used. The computed values of $U_{w}$ using $\alpha=0.14$ as per IEC 61400-3 [4] guidelines and the corresponding sea states calculated with Equation 1 and 2 are presented in Table 2. The load cases in Table 2 have been grouped into 3 scenarios and the probability of occurrence $\left(f_{\text {occ }}\right)$ of the wind speeds within the bounds of each group has been computed and normalized so that they add up to 1 .

Table 2. DLC1.3 metocean data

\begin{tabular}{lllllll}
\hline Scenario & $U_{\text {hub(10min })}$ & $U_{10 \mathrm{~m}(10 \mathrm{~min})}$ & $U_{10 \mathrm{~m}(\mathrm{hhr})}$ & $H_{s}$ & $T_{p}$ & $f_{\text {occ }}$ \\
\hline \multirow{4}{*}{$L C_{1}$} & 4 & 2.94 & 2.79 & 1.94 & 9.73 & \\
& 6 & 4.41 & 4.19 & 2.19 & 9.76 & 0.4069 \\
& 8 & 5.88 & 5.59 & 2.47 & 9.83 & \\
\hline \multirow{4}{*}{$C_{2}$} & 10 & 7.35 & 6.98 & 2.77 & 9.93 & \\
& 12 & 8.82 & 8.38 & 3.10 & 10.06 & \multirow{2}{*}{0.4277} \\
& 14 & 10.29 & 9.78 & 3.44 & 10.21 & \\
& 16 & 11.76 & 11.17 & 3.81 & 10.37 & \\
\hline \multirow{4}{*}{$L C_{3}$} & 18 & 13.23 & 12.57 & 4.19 & 10.54 & \\
& 20 & 14.70 & 13.96 & 4.58 & 10.72 & \multirow{2}{*}{0.1654} \\
& 22 & 16.17 & 15.36 & 4.99 & 10.91 & \\
& 24 & 17.64 & 16.76 & 5.42 & 11.11 & \\
\hline
\end{tabular}


In Table 2, a conversion factor of 0.95 (i.e. the ratio between the 1-hr wind speed and 10-min average wind speed) is used [5]. This adjustment is necessary for simulations lasting 10-min as the 1-hr wind measurement need to be corrected to correspond to the 10-min simulation length. The wind condition is characterized by the extreme turbulence model (ETM). Wind and wave propagation are aligned for DLC1.3 simulations as depicted in Fig. 3 with the effect of yaw misalignment ignored.

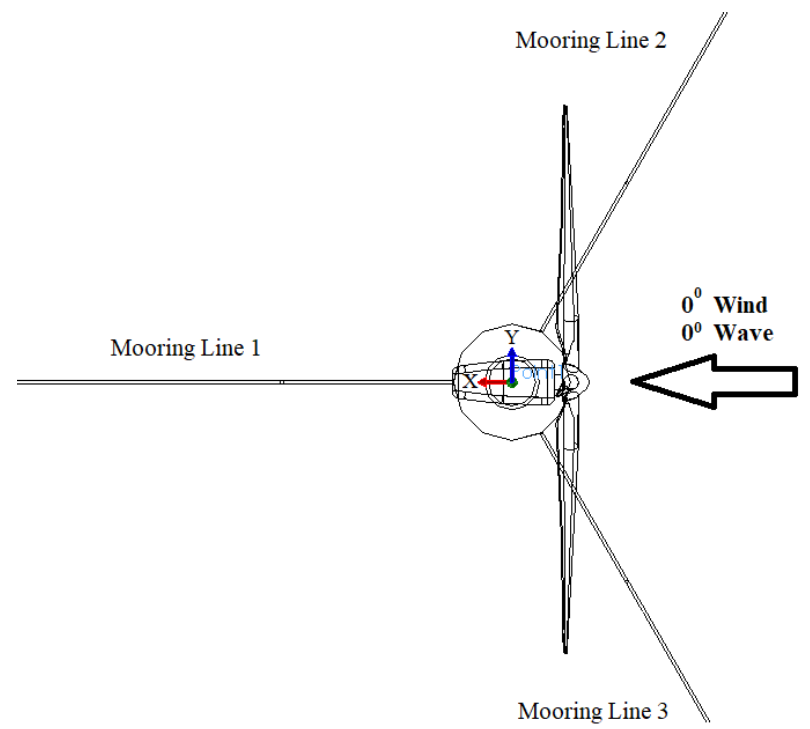

Fig. 3. Bottom view illustration of OC3-Hywind FWT showing DLC1.3 wind/wave direction

DLC1.6a simulates loading resulting from wind conditions characterized by Normal Turbulence Model (NTM) over the power production wind bins in combination with severe sea state (SSS). For the sake of a generic analysis, the SSS is represented by the 50-year wave height $\left(H_{s 50}\right)$ and peak period $\left(T_{p}\right)$ are assumed to be $8.52 \mathrm{~m}$ and $12.45 \mathrm{~s}$.

To replicate a situation where the turbine is shut down to prevent damage due to extreme wind and the rotor is left idling, DLC6.1a is simulated. The blades are feathered at $90^{\circ}$ and all control systems are turned off. The idling scenario is chosen rather than a parked situation (where brakes are applied) as the later is mostly used for maintenance operations. Values corresponding to a recurrence period of 50-years for both wind and waves assumed to occur at the same time are used. The 50-year extreme wind speed at the hub 


$$
\text { w }
$$

height is taken as $41 \mathrm{~m} / \mathrm{s}$. The wind condition is the Extreme Wind Model (EWM) characterized by a turbulence intensity of $11 \%$. For the sea state, the Extreme Sea State (ESS) which is taken as the 50-year wave height and peak period is used $\left(H_{s 50}=8.52 \mathrm{~m}\right.$ and $\left.T_{p}=12.45 \mathrm{~s}\right)$. The influence of windwave misalignment is simulated by applying the mean wind speed at a fixed direction of $0^{\circ}$ while the incident wave direction is varied from $0^{\circ}$ to $345^{\circ}$ with a bin interval of $15^{\circ}$ amounting to 24 bins (see Fig. 4). The simulation

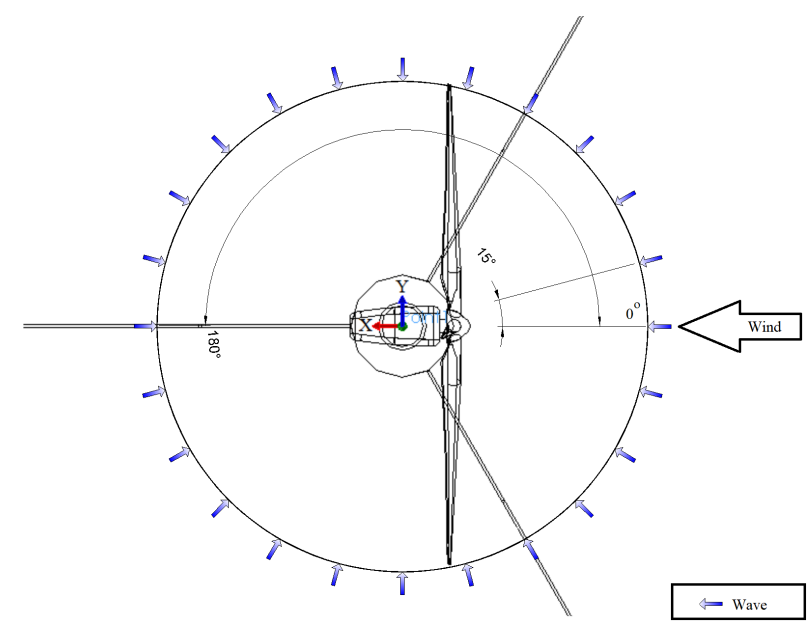

Fig. 4. Bottom view illustration of OC3-Hywind FWT showing DLC6.1a wind/wave misalignment angles

length is 1-hr as per IEC 61400-3 [5]. To capture the stochasticity of the sea state, 6 realizations of wind and wave are used for all DLC simulations. A summary of the DLCs considered in this paper is given in Table 3.

\subsection{Fully coupled time-domain simulation}

To capture the nonlinear dynamic response of the coupled FWT system, the NREL aero-hydro-servo-elastic tool OpenFAST (formerly known as FAST [21]) is used. FAST relies on a combination of modal-dynamics and multibody-dynamics formulation [29]. The underlying theories employed by FAST are not within the scope of this work, readers can refer to Ref. [21, 2931] for details. Each simulation starts with the generation of full-field turbulent wind with Turbsim [32] using Kaimal wind spectrum [4]. The JONSWAP spectrum is used to model the irregular sea waves. The aerodynamic 
Table 3. Summary of DLCs

\begin{tabular}{|c|c|c|c|}
\hline & DLC 1.3 & DLC 1.6a & DLC $6.1 \mathrm{a}$ \\
\hline Wind model & ETM & NTM & EWM \\
\hline Wind speed & $U_{\text {in }}<U_{\text {hub }}<U_{\text {out }}$ & $U_{\text {in }}<U_{\text {hub }}<U_{\text {out }}$ & $U_{h u b}=41 \mathrm{~m} / \mathrm{s}$ \\
\hline Wave Model & NSS & SSS & ESS \\
\hline$H_{s} \mid T_{p}$ & Table 2 & $8.52 m \mid 12.45 s$ & $8.52 m \mid 12.45 s$ \\
\hline Current model & NCM & $\mathrm{NCM}$ & $\mathrm{ECM}$ \\
\hline Current speed & $0.6 \mathrm{~m} / \mathrm{s}$ & $0.6 \mathrm{~m} / \mathrm{s}$ & $1.2 \mathrm{~m} / \mathrm{s}$ \\
\hline Misal & $0^{\circ}$ & $0^{\circ}$ & $0^{\circ}: 15^{\circ}: 345^{\circ}$ \\
\hline Sim.L & $6 \times 10$ min & $6 \times 1 h r$ & $6 \times 1 h r$ \\
\hline
\end{tabular}

loads are calculated with the classical quasi-steady blade element momentum (BEM) theory or the generalized dynamic wake (GDW) model. Wave kinematics are computed using the linear Airy wave theory and the Morison's equation is employed for computing the hydrodynamic loads on the platform. The equations of motion of the multi-bodied turbine system are solved using Kane's dynamics [33]. FAST employs two main control systems in similitude with the style of the Garrad Hassan BLADED wind turbine code [34]. These are a generator-torque controller and a full-span rotor-collective blade pitch controller which are implemented as an external Dynamic Linked Library (DLL). A detailed description of the formulation of the FAST control system can be found in Ref. [29].

\subsection{Determination of design-driving wind bin}

To ascertain design-driving metocean conditions for the selected ULS load cases, several time domain simulations are carried out. For DLC1.3, 6 unique wind and wave realizations for the 11 wind bins in Table 2 are simulated. This amounts to a total of 66 time domain simulations each having a simulation length of $660 \mathrm{~s}$ with the first $60 \mathrm{~s}$ excluded to mitigate the influence of start-up transients. Only a few response channels are presented in Fig. 5 for the sake of brevity. These are the extreme values for 6 unique realizations per wind bin for the tower top longitudinal deflection (YawBrTDxt), tower base fore-aft shear force (TwrBsFxt), tower base fore-aft bending moment (TwrBsMyt), the platform surge (PtfmSurge), tension at the fairleads 2 (FAIRTEN2) and the clearance between the tip of blade 2 and the tower (B2N1Clrnc). In Fig. $5, *$ represents the extreme value of each of the 6 wind/wave seed and the 

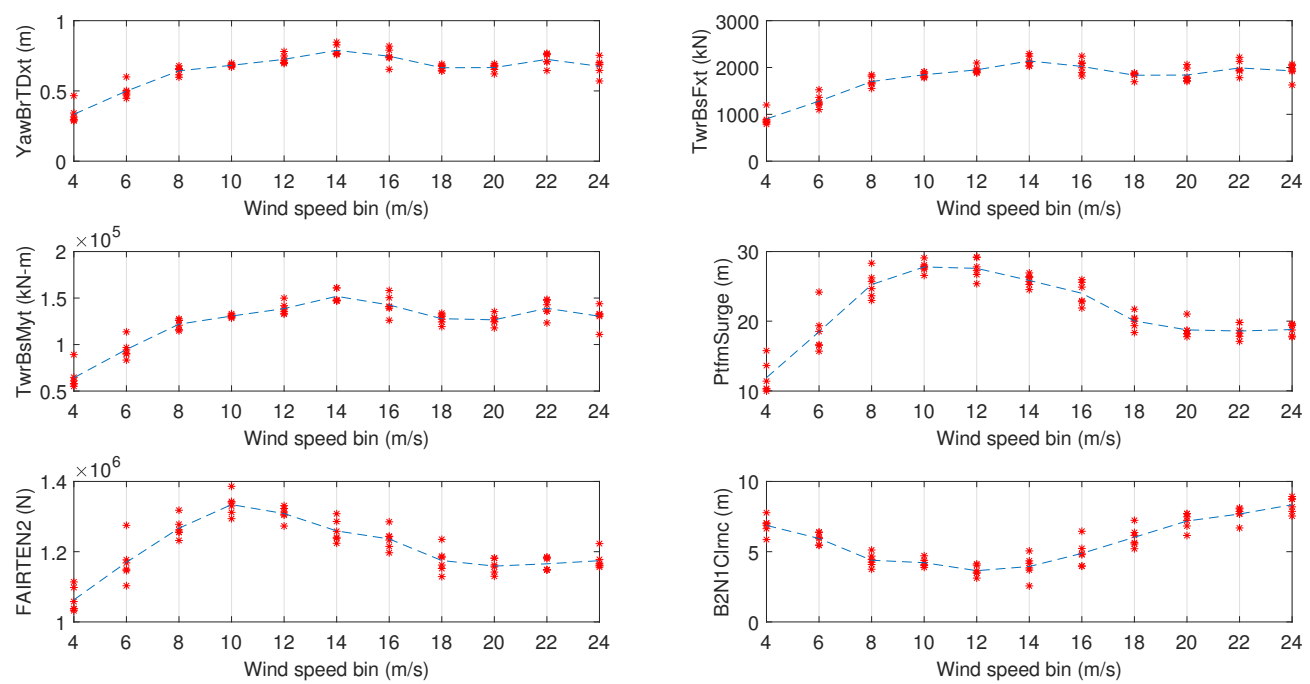

Fig. 5. DLC1.3 turbine responses plotted over wind speed bins

are not presented here) increases with the platform surge excursions. This is clearer by examining Fig. 3 which shows the arrangement of the mooring lines relative to the wind inflow and wave propagation direction. The clearance between the blade tip and the tower is most critical at the $12 \mathrm{~m} / \mathrm{s}$ wind bin. The blade-tip-to-tower clearance presented takes into account the local tower radius, it is however an approximate estimate as it assumes the turbine blade to be a line with no volume. The tower responses such as the deflections, shear forces and moments are most critical for the $8 \mathrm{~m} / \mathrm{s}, 14 \mathrm{~m} / \mathrm{s}$ and $22 \mathrm{~m} / \mathrm{s}$ wind bins within the environmental states of scenario 1,2 and 3 respectively (these bins are also the drivers of maximum von Mises stresses presented in Section 4.2). The bin centres from this section $\left(U_{\text {bin }}^{*}\right)$ are used to train the Kriging models. To account for uncertainty introduced by using a bin interval of $2 \mathrm{~m} / \mathrm{s}$, the trained Kriging models are subsequently used to select the "true" design driving wind bin $\left(U_{b i n}\right)$ to be used in the computation of failure probability - a finer bin interval of $0.1 \mathrm{~m} / \mathrm{s}$ is used (see Section 6.2.2).

A total of 66 simulations were run for DLC1.6a, each lasting $3660 \mathrm{~s}$. Once again the first $60 \mathrm{~s}$ is expunged from the response statistics. The results are presented in Fig. 6. From the response channels examined (including those not presented), most of the extreme events occur when the wind speed is around the rated wind speed of $11.4 \mathrm{~m} / \mathrm{s}$. This is attributable to the influence of the action of the control system. It is clear that the design driving wind bin for the tower is the $12 \mathrm{~m} / \mathrm{s}$ wind bin. As with DLC1.3, the design driving 

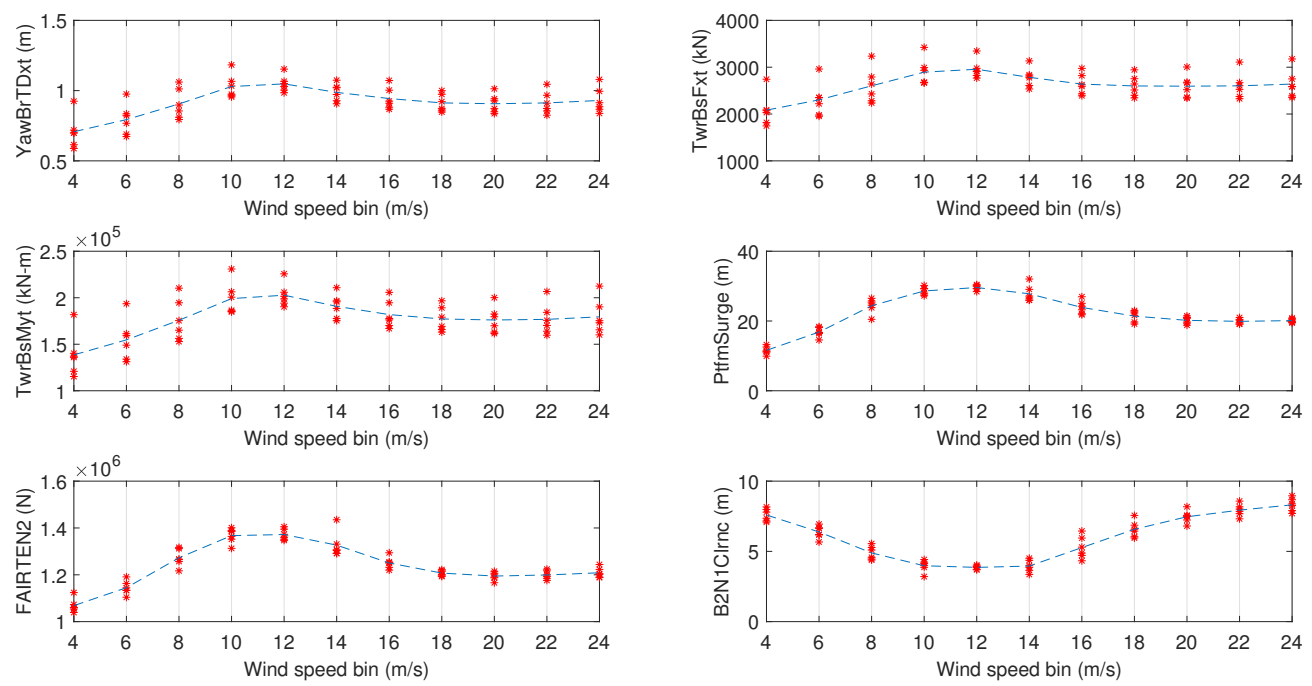

Fig. 6. DLC1.6a turbine responses plotted over wind speed bins

bin at this point for DLC1.6a $(12 \mathrm{~m} / \mathrm{s})$ is used for training the Kriging model which is subsequently used to select the "true" design driving wind bin (see Section 6.2.2).

Presented in Fig. 7 are rose plots for responses covering DLC6.1a bins. A total of $24 \times 6$ simulations of $3660 \mathrm{~s}$ long were carried out. The results presented are the mean values from 6 unique wind/wave realizations. The
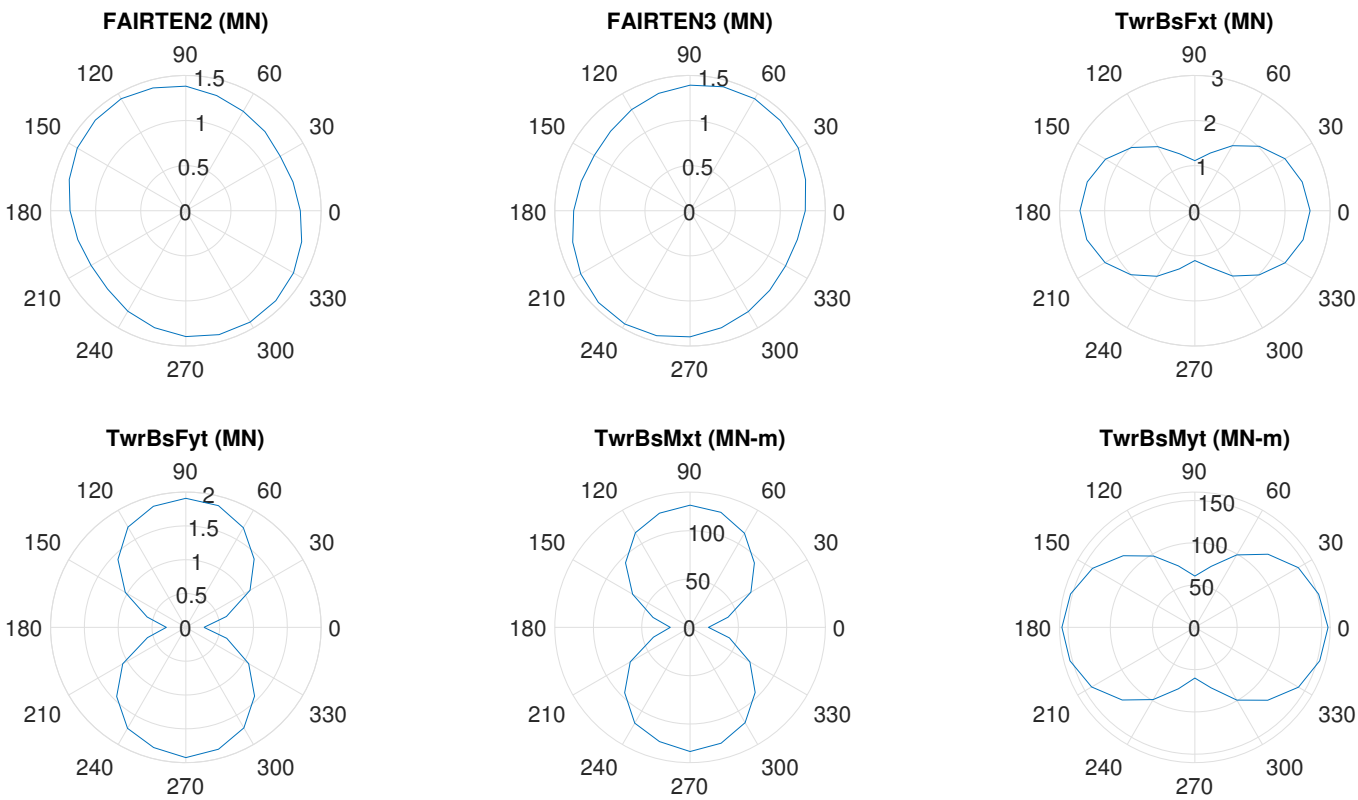

Fig. 7. Variation of load channels with wind-wave misalignment 
collinear and perpendicular wind/wave misalignment bins produced the most severe tower loads (see Fig. 7). This is reflective of the axisymmetric design of the spar. In terms of load effect like tower von Mises stress, it is not clear what bin would result in the most stresses in the tower. This is addressed in Section 4.2. The maximum tension at fairlead 2 occurs when the wind/wave misalignment is $120^{\circ}$ while a misalignment of $240^{\circ}$ causes maximum tension at the fairlead 3.

\section{Load effect computation from aero-hydro-servo-elastic simula- tion}

\subsection{Finite Element structural stress analysis}

Structural stress analysis usually require finite element (FE) simulations. Given that the loading to be transferred to an FE model come in time series, evaluating the stress state using a yield criterion like von Mises stress at every time step imposes huge computational burden. To address this issue, the use of a linear relationship between applied loads and the nodal/element displacements, strains and stresses is investigated in this section.

\subsubsection{Finite Element model}

The tower is a vital structural member of the support structure. It links the Rotor-Nacelle-Assembly (RNA) to the platform. Stress analysis is performed using the FE solver, Abaqus. The tower is modelled with shell elements since it can be classed as a thin-walled structural member. A fixed boundary condition is applied to the tower base. The tower experiences stresses due to deflections resulting from platform motions and imposed loads. The tower top forces and moments emanate from wind loading over the rotor, inertial forces from structural dynamics as well as the weight of the RNA. Along its span, inertial forces, the weight of the tower and distributed wind loading on the tower are eminent. FAST outputs 6 component loads at the tower top i.e. 3 forces in $x, y$, and $z$ directions (TFx, TFy and TFz) and moments about the $x, y$, and $z$ axes (TMx TMy, and TMz). In the FE model, these 6 component loads are applied to the tower top nodes by means of a tie connection between a rigid nacelle base plate and the tower top nodes of the FE tower. The tower is partitioned into 10 sections for the application of appropriate loads acting over these sections. These comprises inertial forces of each section from the global structural dynamics, weight of each section and wind drag forces acting on each section. FAST can output lumped loads 
at strain gauge locations along the tower. These strain gauge outputs are a summation of all loads acting above respective strain gauge locations. The contribution of each section $n$, equal to $1,2, . ., 10$ (numbered from the tower top) are calculated with Equation 4.

$$
T w F_{n}= \begin{cases}T w H t F_{n}-T F & \text { for } n=1 \\ T w H t F_{n}-T w H t F_{n-1} & \text { for } n=2,3, . ., 9 \\ T w r B s F-T w H t F_{n} & \text { for } n=10\end{cases}
$$

where $T w F_{n}$ is resultant forces acting only on section $n, T w H t F_{n}$ is the lumped loads summed up at strain gauge $n, T F$ is the tower top forces and TwrBsF is the tower base forces. All forces have $x, y$ and $z$ components. These 3 component forces for each section are applied as body forces to corresponding sections in Abaqus $\left(B F n=T w F_{n} \times V_{n}\right.$. $V_{n}$ is the volume of tower section $n$ ). The tower top loads and sectional body forces (a total of 36 load components) at each time step are written as load amplitude tables and applied to the Abaqus model. The time series of tower base reaction forces computed by Abaqus matches those computed by FAST. This is however computationally prohibitive given the large number of tower elements (5280 elements). To speed up the computation, the method used by Wandji et al. [15] in the stress analysis of a universal joint for a combined monopile and spar-buoy floater concept is adopted and extended. The method is hinged on the principle of linear elasticity. Under the typical tower loading conditions, nodal displacements, element strains and stresses can be expressed as a linear combination of the applied FAST loads according to Equation 5. 


$$
R=\left[K_{R, 1}, K_{R, 2}, \cdots, K_{R, 36}\right]\left[\begin{array}{cccc}
T F x_{t_{0}} & T F x_{t_{0}+\Delta t} & \cdots & T F x_{t_{\max }} \\
T F y_{t_{0}} & T F y_{t_{0}+\Delta t} & \cdots & T F y_{t_{\max }} \\
T F z_{t_{0}} & T F z_{t_{0}+\Delta t} & \cdots & T F z_{t_{\max }} \\
T M x_{t_{0}} & T M x_{t_{0}+\Delta t} & \cdots & T M x_{t_{\max }} \\
T M y_{t_{0}} & T M y_{t_{0}+\Delta t} & \cdots & T M y_{t_{\max }} \\
T M z_{t_{0}} & T M z_{t_{0}+\Delta t} & \cdots & T M z_{t_{\max }} \\
B F 1 x_{t_{0}} & B F 1 x_{t_{0}+\Delta t} & \cdots & B F 1 x_{t_{\max }} \\
B F 1 y_{t_{0}} & B F 1 y_{t_{0}+\Delta t} & \cdots & B F 1 y_{t_{\max }} \\
B F 1 z_{t_{0}} & B F 1 z_{t_{0}+\Delta t} & \cdots & B F 1 z_{t_{\max }} \\
\vdots & \vdots & \cdots & \vdots \\
B F 10 x_{t_{0}} & B F 10 x_{t_{0}+\Delta t} & \cdots & B F 10 x_{t_{\max }} \\
B F 10 y_{t_{0}} & B F 10 y_{t_{0}+\Delta t} & \cdots & B F 10 y_{t_{\max }} \\
B F 10 z_{t_{0}} & B F 10 z_{t_{0}+\Delta t} & \cdots & B F 10 z_{t_{\max }}
\end{array}\right]
$$

where:

$R=U T, \epsilon_{i j}(i j=11,22,12), \sigma_{i j}(i j=11,22,12)$

$U T=$ nodal translation

$\epsilon_{i j}(i j=11,22,12)=$ strain components

$\sigma_{i j}(i j=11,22,12)=$ stress components

$t_{0}, \Delta t$ and $t_{\max }$ are the FAST time series start time, time-step and end time respectively. The coefficients $K_{R, 1}, K_{R, 2}, \cdots, K_{R, 36}$ are obtained by running FE simulation in Abaqus for unit-load cases for each load input while other loads are set to zero. In other words the big matrix on the right of Equation 5 becomes a $36 \times 36$ diagonal matrix with all elements in the diagonal set as 1 , this is then used as load amplitude table in Abaqus simulation. For each node/element, the resulting $R$ vector becomes the coefficients. Estimating the tower element stresses for any given FAST time series becomes a trivial matrix operation given by Equation 5. Under plane stress conditions (i.e. stress components $\sigma_{3}=0$ and $\sigma_{23}=\sigma_{31}=0$ ), the von Mises stress $\sigma_{v}$ can then be computed using Equation 6 .

$$
\sigma_{v}=\sqrt{\sigma_{11}^{2}+\sigma_{11} \sigma_{22}+\sigma_{22}^{2}+3 \sigma_{12}^{2}}
$$

To validate the linearization described by Equation 5, a comparison between the time series of the longitudinal and lateral tower top deflections 

concentrations easily identifiable.
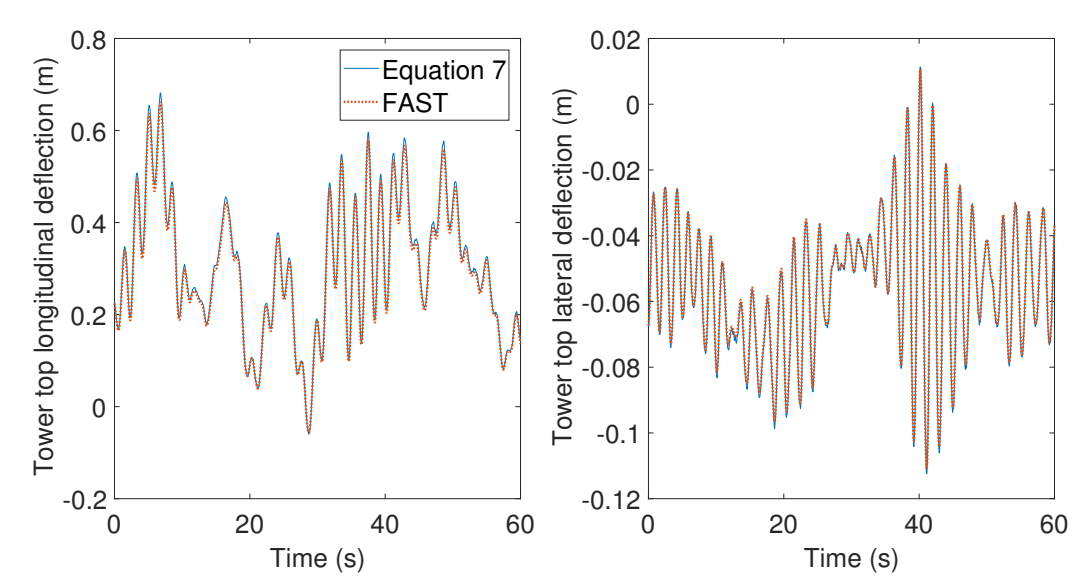

Fig. 8. Displacement of tower top node

outputted by FAST and those computed using Equation 5 is presented in Fig. 8. The computed Normalized Root Mean Squared Error (NRMSE) was around 0.03 and -0.02 for longitudinal and lateral deflections respectively for $600 \mathrm{~s}$ time series. The 3 -D FE tower model captures more modes and eigenfrequencies than the beam representation employed by FAST (four modes). Contribution from these modes would no doubt cause some disparity in the deflections of the different tower models. This level of agreement is a cogent pointer to the validity of the approach presented. A further comparison is made between the stresses obtained using computationally expensive Abaqus simulations and stresses obtained with Equation 5 for a single element in the tower base is shown in Fig. 10. The results show that the linearization gives values that closely match Abaqus results with NRMSE around $10^{-5}$. It takes only about $0.06 \mathrm{~s}$ to evaluate a $600 \mathrm{~s}$ long time series for tower top deflections and about $0.3 \mathrm{~s}$ for the time series of maximum von Mises stress in the tower (Fig. 9a shows a time series of maximum von Mises stress in the tower). A stress contour plot of a single time step is shown in Fig. 9b, with local stress

\subsection{Ranking Design load cases based on load effect}

To rank the DLCs based on load effects, not just the load channels outputted from FAST should be used but also computed load effect such as stresses in structural members. The stress state of the structural components is key in reliability analysis or optimization exercises. The load effect 


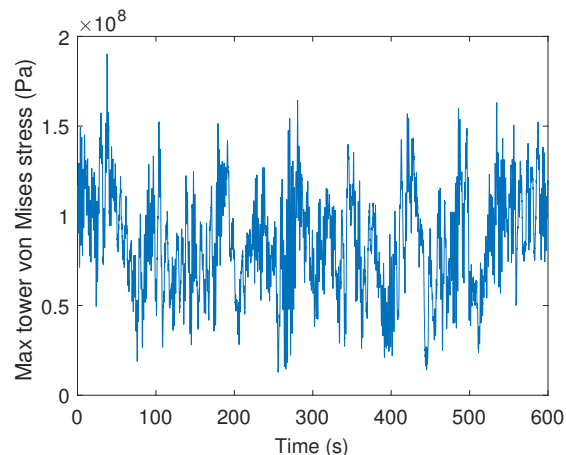

(a) Von Mises stress time series

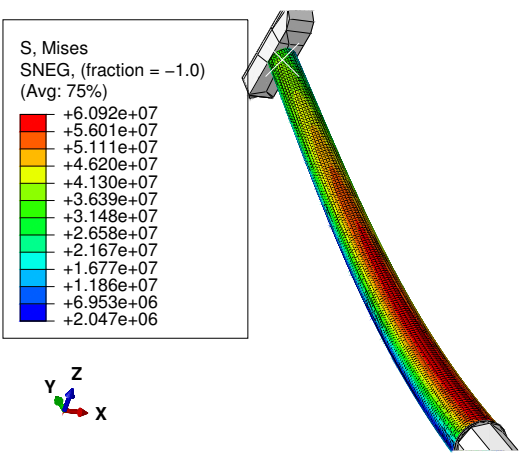

(b) von Mises Stress contour plot

Fig. 9. Tower von Mises stress
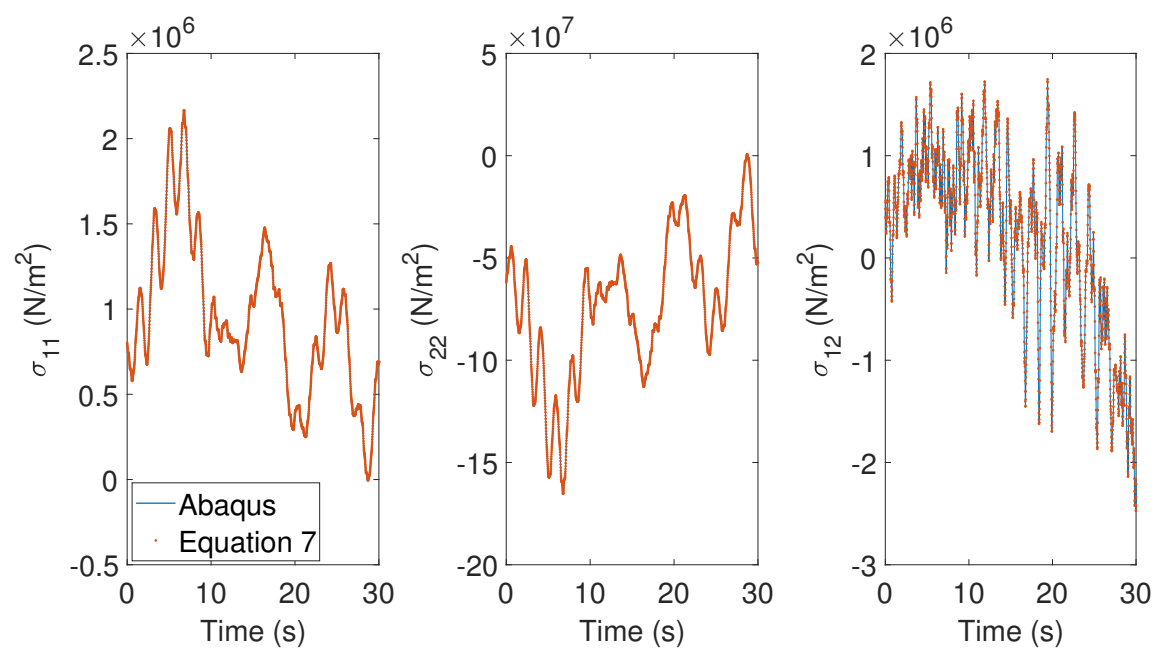

Fig. 10. Comparison between Abaqus simulation and linearization of stress components

in terms of von Mises stress becomes trivial to evaluate for all DLC bins using the methodology presented in Section 4.1.1. The results are shown in Fig. 11a, 11b and 12 for DLC1.3, 1.6a and 6.1a respectively. This implies huge computational savings in estimating the stress state of structural members under linear elastic loading from time series of aero-hydro-servo-elastic simulations.

From Fig. 12, it is clear that the extreme von Mises stress on the tower for DLC6.1a occurs when the wind and wave are collinear wih a $0^{\circ}$ misalingnment. Combining the results from select load channels and stress eval- 


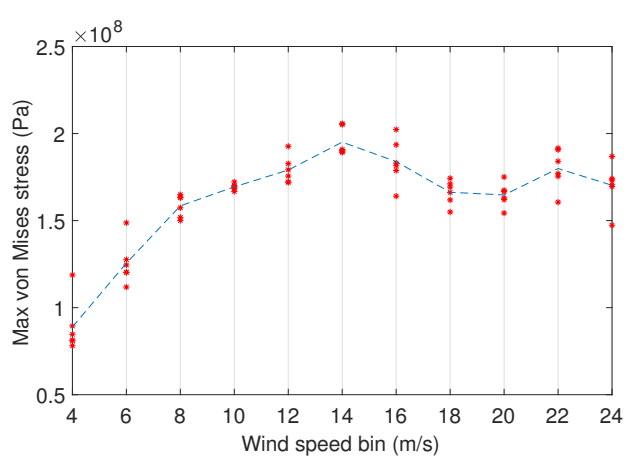

(a) DLC1.3 wind bins

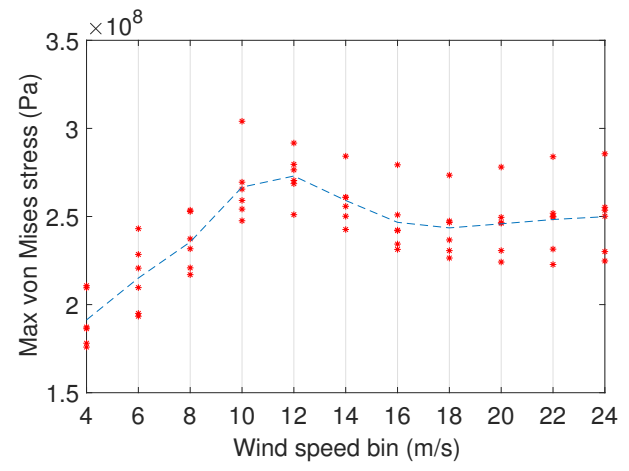

(b) DLC1.6a wind bins

Fig. 11. Tower von Mises stress plotted over wind bins von Mises Stress (MPa)

90

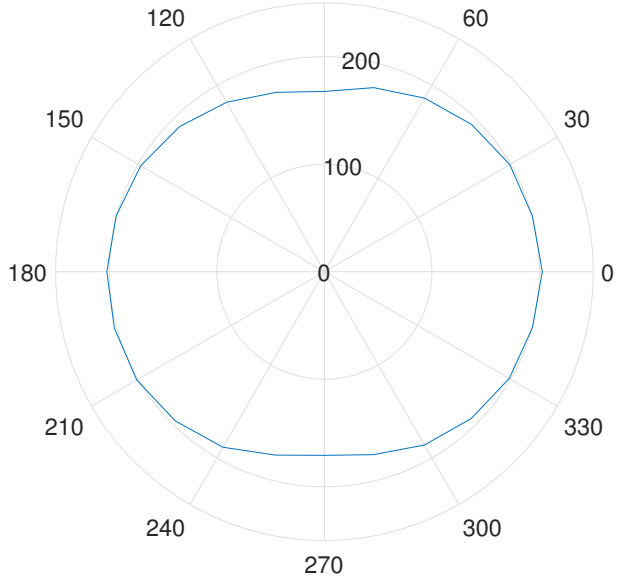

Fig. 12. Variation of tower von Mises stress for DLC6.1a bins

uations for all the DLCs, ranking of the DLCs is presented in Fig. 13. The results presented in Fig. 13 are the maximum between the tower top displacements (TTDxy), tower base shear forces (TBFxyt) and tower base moments (TBMxyt) in $x$ and $y$ directions. Also presented are the extreme von Mises stress observed in the tower (TWR-VM), the maximum platform surge and pitch displacements as well as the maximum observed tension in all three fairleads. The minimum of the blade to tower clearance for all three blades is reported as $\mathrm{BN} 1 \mathrm{Cl}$. For ease of comparison, the results have been normalized by the maximum in all DLCs for each load channel except for $\mathrm{BN} 1 \mathrm{Cl}$ where the values have been normalized by the minimum and then inverted. 


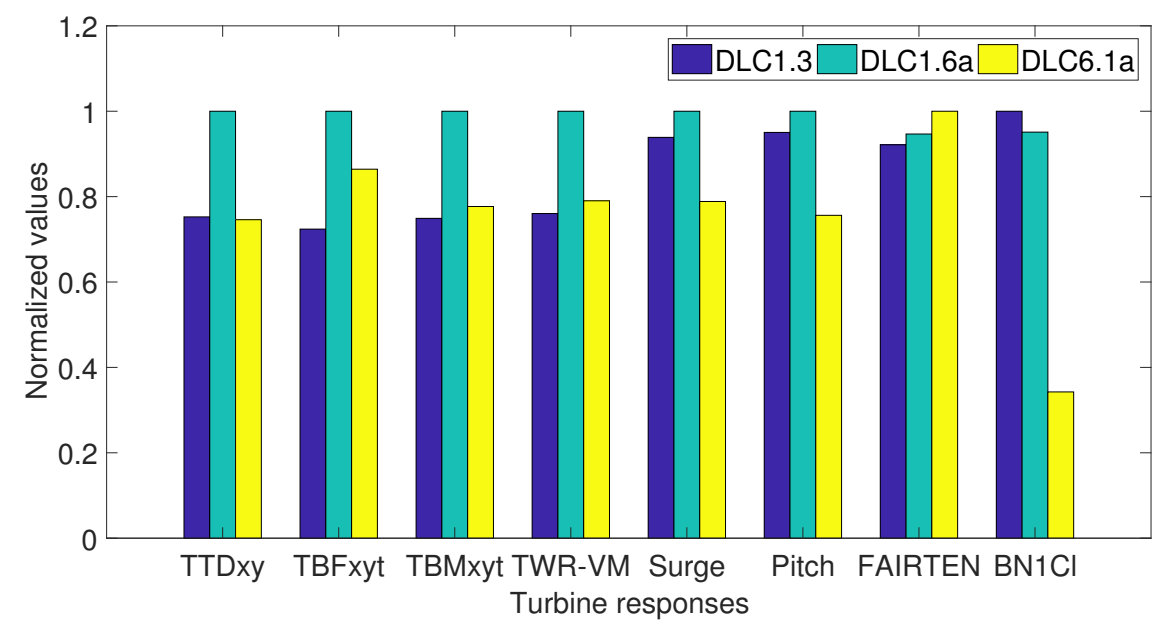

Fig. 13. Ranking DLCs based on design responses

Most of the extreme responses occur with DLC1.6a conditions. This is due to the combined action of severe sea state, rotor dynamics and loads from the working of the turbine controllers. The maximum fairlead tension is observed for DLC6.1a as wind/wave misalignment was considered for this load case. For the blade-tip to tower clearance, DLC1.3 with ETM has the most critical value closely followed by DLC1.6a. It will suffice to posit that the DLC1.6a amounts to extreme loads for tower design out of the three load cases considered.

\section{Surrogate Modeling of Ultimate Loads}

The design of FWT support structures requires reliability analysis and optimization exercises. Thousands if not millions of evaluation of implicitly defined LSFs is needed. This no doubt can be computationally expensive if not prohibitive. A solution to this problem is the use of surrogate models or metamodels. Surrogate models are created by constructing a relationship between a relatively few set of input variables and their corresponding responses (generated by running the original computationally expensive model). By so doing, the implicitly defined LSFs become explicitly defined in terms of the input variables. 


\subsection{Design Space and Input Domain}

To effectively capture the variability of the system using relatively few sample points, it is important that the design space has an efficient spatial spread of the distributions of the random variables used in building the surrogate models. To this end, LHS is employed. LHS works by dividing the subspace of each input variable $x_{i} ; i=1,2, . ., N$ into $S$ disjoint subsets having equal probability $\Omega_{i k}, i=1,2, . ., N, k=1,2, . ., S$. For each input variable, samples are drawn from the various strata according to: $X_{i k}=D_{x_{i}}^{-1}\left(U_{i k}\right)$, where $D_{x_{i}}(\cdot)$ is the marginal cumulative distribution function (CDF) of variable $x_{i}, U_{i k}$ are independent and identically uniformly distributed samples on $\left[\frac{k-1}{S}, \frac{k}{S}\right]$. Finally permutation of the generated sample vectors is done to form the sample points [35]. Table 4 shows the random and deterministic variables used in this study.

The sea state for DLC1.3 is not included in Table 4 , as $H_{s}$ and $T_{p}$ are conditioned on the mean wind speed. This accounts for the correlation between mean wind speed and sea state as described in Section 3.3. For each sample point with a mean wind speed $U_{h u b}$, the equivalent $1 \mathrm{hr}$ wind speed at $10 \mathrm{~m}$ above SWL $\left(U_{10 m}\right)$ is calculated, then the corresponding sea state characterized by $E\left[H_{s} \mid U_{10 m}\right]$ and $E\left[T_{p} \mid U_{10 m}, H_{s}\right]$ are computed.

\subsection{Kriging metamodel}

Kriging is a statistical interpolation method based on Gaussian process modeling. It was originally introduced in the field of geostatistics by Matheron [37]. Kriging has since been applied to various fields such as computer experiments [38], structural reliability problem [39] and is gaining popularity in many other fields. The Kriging methodology relies on linear weights that account for data closeness, redundancy and spatial continuity. These weights are unbiased and minimize the estimation variance, thus Kriging is commonly referred to as the best linear unbiased estimator. Kriging predicts the value of outputs $Y(\boldsymbol{x})$ which are computationally expensive to evaluate using the sum of the weighted values of surrounding sample points $\boldsymbol{x}=x_{1} \ldots x_{k}$ obtained from experiments or complex numerical simulations. The Kriging estimator is described by Equation 7 .

$$
Y^{*}(\boldsymbol{x})=\boldsymbol{\beta}^{T} \boldsymbol{f}(\boldsymbol{x})+Z(\boldsymbol{x})
$$


Table 4. Random and deterministic variables used for surrogate model training and reliability analysis $[10,15,16,36]$

\begin{tabular}{|c|c|c|c|}
\hline Parameter & Dist. & Mean & $\mathrm{CoV}$ \\
\hline DLC1.3 wind speed, $U_{h u b}^{*}(\mathrm{~m} / \mathrm{s})$ & $\mathrm{N}$ & $8,14,22$ & 0.05 \\
\hline DLC1.6a Wind speed, $U_{h u b}^{*}(\mathrm{~m} / \mathrm{s})$ & $\mathrm{N}$ & 12 & 0.05 \\
\hline DLC1.6a Seastate, $H_{s}^{*}(m) \mid T_{p}^{*}(s)$ & $\mathrm{N}$ & $8.52 \mid 12.45$ & 0.05 \\
\hline DLC6.1a wind speed, $U_{h u b}^{*}(\mathrm{~m} / \mathrm{s})$ & $\mathrm{N}$ & 41 & 0.05 \\
\hline DLC6.1a Seastate, $H_{s}^{*}(m) \mid T_{p}^{*}(s)$ & $\mathrm{N}$ & $8.52 \mid 12.45$ & 0.05 \\
\hline Young's modulus, $E^{*}(G P a)$ & $\mathrm{N}$ & 210 & 0.05 \\
\hline Yield stress, $F_{y}(M P a)$ & $\mathrm{LN}$ & 355 & 0.05 \\
\hline Mooring breaking load, $B_{L}(M N)$ & LN & 6.65 & 0.05 \\
\hline Tower density, $\rho_{t}^{*}\left(\mathrm{~kg} / \mathrm{m}^{3}\right)$ & $\mathrm{N}$ & 8500 & 0.05 \\
\hline Tower base thickness, $t_{t}^{*}(m)$ & $\mathrm{N}$ & 0.027 & 0.03 \\
\hline Tower base outside diameter, $D(m)$ & & 6.5 & \\
\hline Yield model uncertainty, $X_{y}$ & $\mathrm{LN}$ & 1 & 0.05 \\
\hline Kriging model, $X_{k r i g}$ & $\mathrm{LN}$ & 1 & Table 5 \\
\hline Exposure (terrain), $X_{\text {exp }}$ & $\mathrm{LN}$ & 1 & 0.10 \\
\hline Structural dynamics, $X_{d y n}$ & $\mathrm{LN}$ & 1 & 0.05 \\
\hline Aerodynamic parameters, $X_{\text {aero }}$ & $\mathrm{LN}$ & 1 & 0.10 \\
\hline Hydrodynamic parameters, $X_{h y d r o}$ & $\mathrm{LN}$ & 1 & 0.10 \\
\hline Load effect computation, $X_{\text {str }}$ & $\mathrm{N}$ & 1 & 0.03 \\
\hline
\end{tabular}

Dist.: Distribution; *: Variables for Kriging model; N: Normal;

LN: Lognormal; CoV: Coefficient of variation

where $Y^{*}(\boldsymbol{x})$ is the Kriging estimate. The first term in Equation 7 is the mean value or trend of the output consisting of $N$ basis functions $f_{i} ; i=1, \ldots, N$ and corresponding regression coefficients $\beta_{i} ; i=1, \ldots, N$. Given in Equation8 and 9, are the trends for the ordinary Kriging and universal Kriging metamodels respectively. The simple Kriging is not covered for sake of brevity.

$$
\begin{gathered}
\boldsymbol{\beta}^{T} \boldsymbol{f}(\boldsymbol{x})=\beta_{0} \\
\boldsymbol{\beta}^{T} \boldsymbol{f}(\boldsymbol{x})=\sum_{t=0}^{N} \beta_{t} f_{t}(x)
\end{gathered}
$$


In the ordinary Kriging, the trend has a constant but unknown value. For universal Kriging, the trend is assumed to be a linear combination of arbitrary functions which can be linear, quadratic or any polynomial. The performance of ordinary, linear and quadratic Kriging is presented in Section 5.3. The second term in Equation 7 represents the Gaussian process described by a zero mean, variance $\sigma^{2}$ and covariance given by Equation 10 .

$$
\operatorname{Cov}\left(\boldsymbol{x}, \boldsymbol{x}^{\prime}\right)=\sigma^{2} R\left(\boldsymbol{x}, \boldsymbol{x}^{\prime}, \boldsymbol{\theta}\right)
$$

where $R$ represents the correlation function having associated hyperparameters $\boldsymbol{\theta}$. The correlation function $R$ describes the correlation between $\boldsymbol{x}$ and $\boldsymbol{x}^{\prime}$.

The Kriging module contained in the framework for uncertainty quantification toolbox developed by UQLab [40], is used in this study. The toolbox provides options for optimization of Kriging hyper-parameters. Readers can refer to Ref. [40] for details. In order to select a suitable Kriging model, the set of hyper-parameters $\boldsymbol{\sigma}^{2}, \boldsymbol{\beta}$ and $\boldsymbol{\theta}$ that maximizes the likelihood of observations are estimated using maximum likelihood method for different trends. The choice of appropriate trend, correlation function and sample size is a key challenge in calibration of the Kriging model. A combinatorial method similar to those employed by Ref. [7] is adopted in this paper.

\subsection{Kriging calibration and sample size sensitivity}

Selecting the optimal combination of the trend and correlation function of a Kriging model can be quite a challenge. To address this challenge, a comparison is made between Ordinary Kriging and universal Kriging (linear and quadratic trends) used in combination with Matérn-3/2, Matérn-5/2 and exponential correlation functions. The sample points are obtained from DLC1.3 aero-hydro-servo-elastic simulations. For each combination, the best Kriging model is selected after 5 training iterations using the minimum observed NRMSE given by Equation 11 as the basis for selection and also for comparing model performance.

$$
N R M S E=\frac{\sqrt{\frac{1}{p} \sum_{k=1}^{p}\left(Y_{k}-Y_{k}^{*}\right)^{2}}}{\frac{1}{p} \sum_{k=1}^{p}\left(Y_{k}\right)}
$$


where $p$ is the number of validation or test points, $Y$ and $Y^{*}$ are the actual values and Kriging predictions respectively. Apart from the trend and correlation function selected, the number of sample points used in training the Kriging model also have significant effect on the accuracy of the model predictions. Generally, it is the aim to achieve good predictions with minimal samples as evaluation of large sample points can be computationally expensive if not prohibitive. A compromise between computational cost and prediction error has to be made even though increasing the number of sample points generally improves the accuracy of prediction.

We use four sample sizes, $M=[50,100,150,200]$ to investigate Kriging sample size sensitivity and calibration of Kriging model. An additional 50 samples is used as the validation set for model selection from 5 training recursions, while the generalization capability of the models is checked with an independent test set of 250 samples. For each sample point, 6 unique wind/wave random seeds is simulated, this gives a total of 4800 DLC1.3 stochastic simulations. The design load is taken as the mean of the extreme values for the 6 wind/wave realizations, representing the outputs of the simulations for each load channel. The variation of NRMSE computed for the test set of 250 sample points for various Kriging models ( 9 combinations of trend and correlation function) is presented in Fig. 14 for various response channels. The influence of sample size is also shown in Fig. 14. The results presented in Fig. 14 show that the choice of correlation function and trend is affected not only by the sample size but also by the response been modeled. For response (a) and (b), the linear trend with a Matérn-3/2 correlation function performed better overall. This was closely followed by the linear trend with an exponential correlation function. For response (c), the quadratic trend with a Matérn-3/2 gave best results on average while the linear trend with an exponential function performed best considering response (d). It will suffice to say that selection of trend and correlation function is dependant on the nature of the data been modeled. Sample size also affects the accuracy of the Kriging model as seen in Fig. 14. The NRMSE generally reduces with larger sample size especially in response (a) and (b). Other factors such as the quality of the experimental design can influence the generalization capability of the Kriging model. When the experimental design does not have a sufficient spread of the distribution, the generalization of the model can be effected irrespective of sample size. Possible improvements to LHS are contained in literature such as [41], and were not investigated in this paper. The same combinatorial approach is used for DLC1.6a and 6.1a (see Fig. 

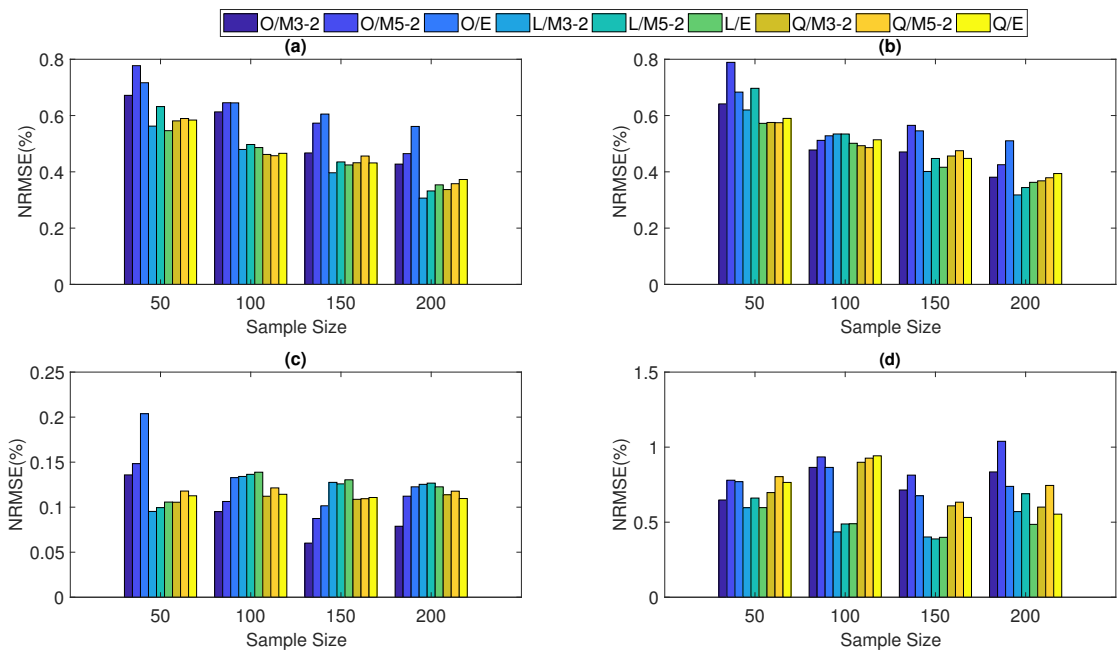

Fig. 14. DLC1.3 variation of NRMSE for (a) Maximum Von Mises stress in tower, (b) Tower base fore-aft bending moment, (c) Maximum fairlead Tension and (d) Minimum blade tip clearance.

O: Ordinary Kriging, L: Linear Trend, Q: Quadratic trend, M3-2: Matérn-3/2, M5-2: Matérn-5/2, E : Exponential

15 and 16 respectively). These DLCs require $1 h r$ long simulations making the evaluation of numerous experimental points computationally expensive. As such, only 100 sample points are used as training set while 50 sample points are used for validation and model selection. The results in Fig. 15 show that the linear trend with an exponential correlation function performed better in DLC1.6a responses except in response (c) where the linear trend and a Matérn-3/2 performed better. In Fig. 16 for DLC6.1a, the linear trend in combination with an exponential correlation function performed better for most of the responses considered except for response (a) where it was out performed by the linear trend with a Matérn-3/2 correlation function. We posit from these results that the selection of appropriate trend and correlation function depends not only on the DLC been modelled but also on the response channel and as such a combinatorial approach is recommended to select the appropriate parameters for a given response and DLC.

\subsection{Accuracy of Kriging Predictions}

Using the trained Kriging models, a one to one comparison between the Kriging predictions and the test data is presented in Fig. 17, 18 and 19. Also 

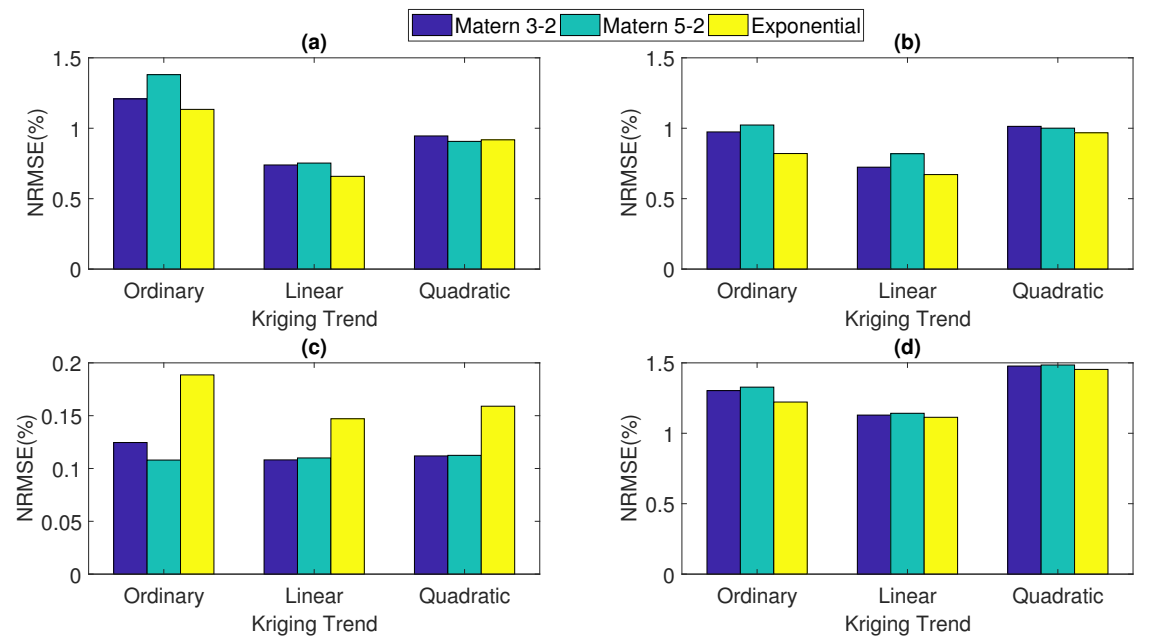

Fig. 15. DLC1.6a variation of NRMSE with different trend and correlation functions for (a) Maximum Von Mises stress in tower, (b) Tower base fore-aft bending moment, (c) Maximum fairlead Tension and (d) Minimum blade tip clearance
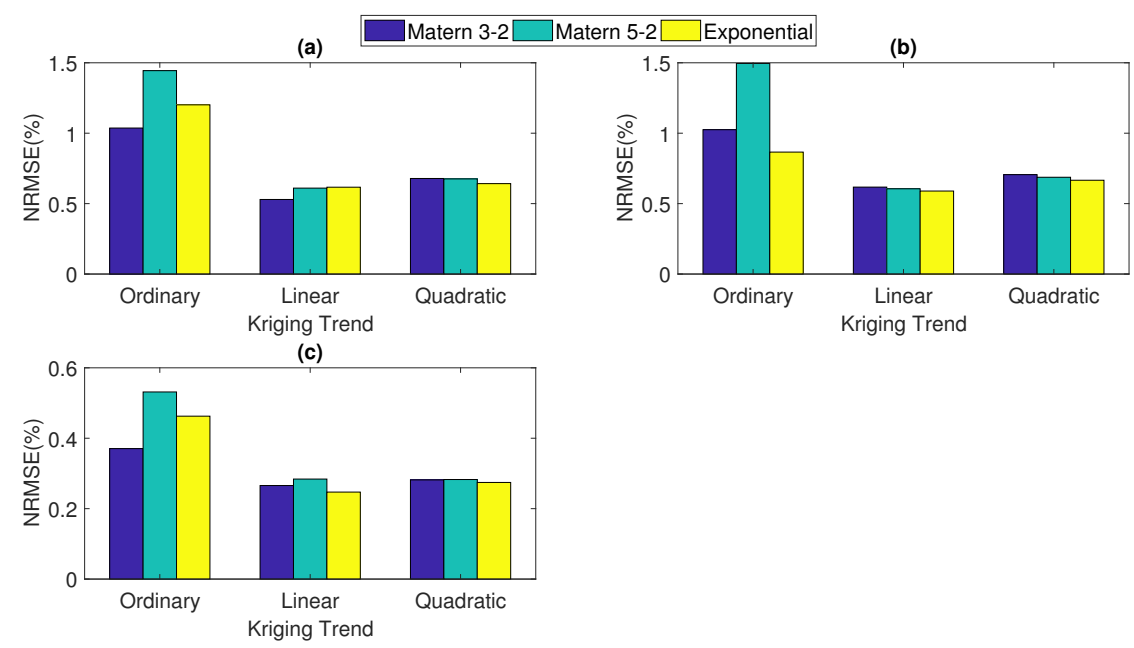

Fig. 16. DLC6.1a variation of NRMSE with different trend and correlation functions for (a) Maximum Von Mises stress in tower, (b) Tower base fore-aft bending moment, (c) Maximum fairlead Tension

included are the Coefficient of Determination $\left(R^{2}\right)$, computed according to Equation 12. 


$$
R^{2}=1-\frac{\sum_{k=1}^{p}\left(Y_{k}-Y_{k}^{*}\right)^{2}}{\sum_{k=1}^{p}\left(Y_{k}-\frac{1}{p} \sum_{k=1}^{p} Y_{k}\right)^{2}}
$$

The R-squared measures the closeness of the target data to the surrogate model predictions. For the considered responses, the Kriging model explains about $93 \%-98.9 \%$ of the variability in the turbine responses considered for DLC1.3 as seen in Fig. 17. The predictions for DLC1.6a and 6.1a in Fig. 18 and 19 respectively, show $R^{2}$ values ranging from $94.8 \%-99.4 \%$. This is a demonstration of the validity of a well calibrated Kriging model for predicting the responses of FWT substructure.
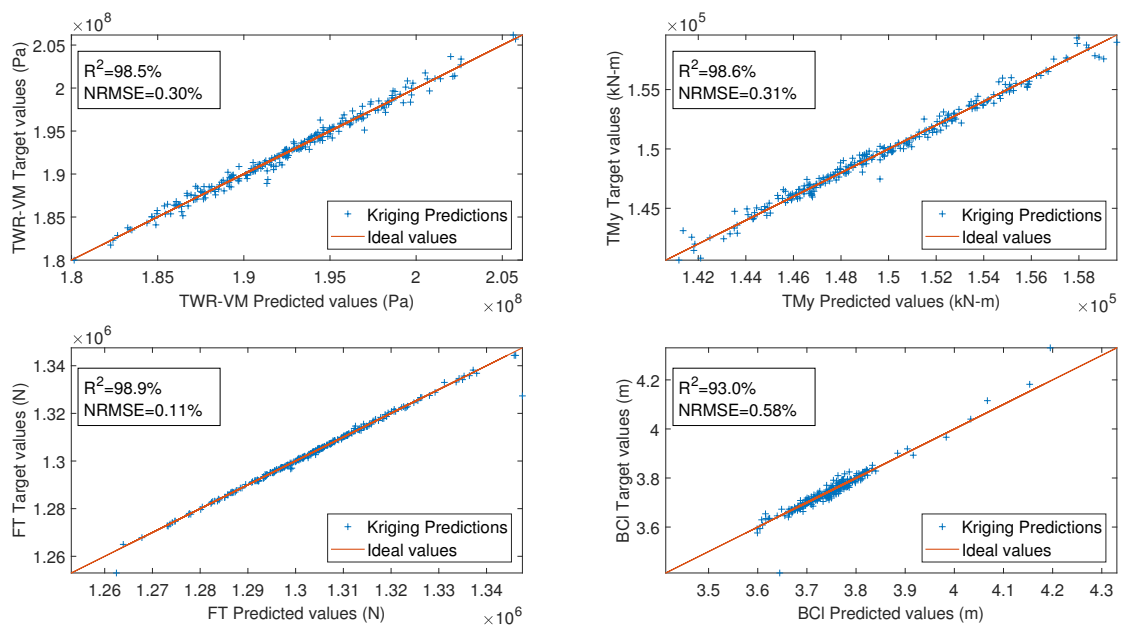

Fig. 17. DLC1.3 Kriging predictions Vs. Target values

\subsection{Characterization of Kriging model' uncertainty}

To estimate the uncertainty of the Kriging model, the procedure outlined in Annex D8.2.2 of Eurocode 1990 [12] is used. This approach was also employed in Ref. [8, 42]. For each load case, 50 sample points are employed for estimating the Kriging uncertainty. The turbine load is first represented by a probabilistic model given by Equation 13 .

$$
L=b_{K} \cdot L_{K} \cdot \epsilon
$$



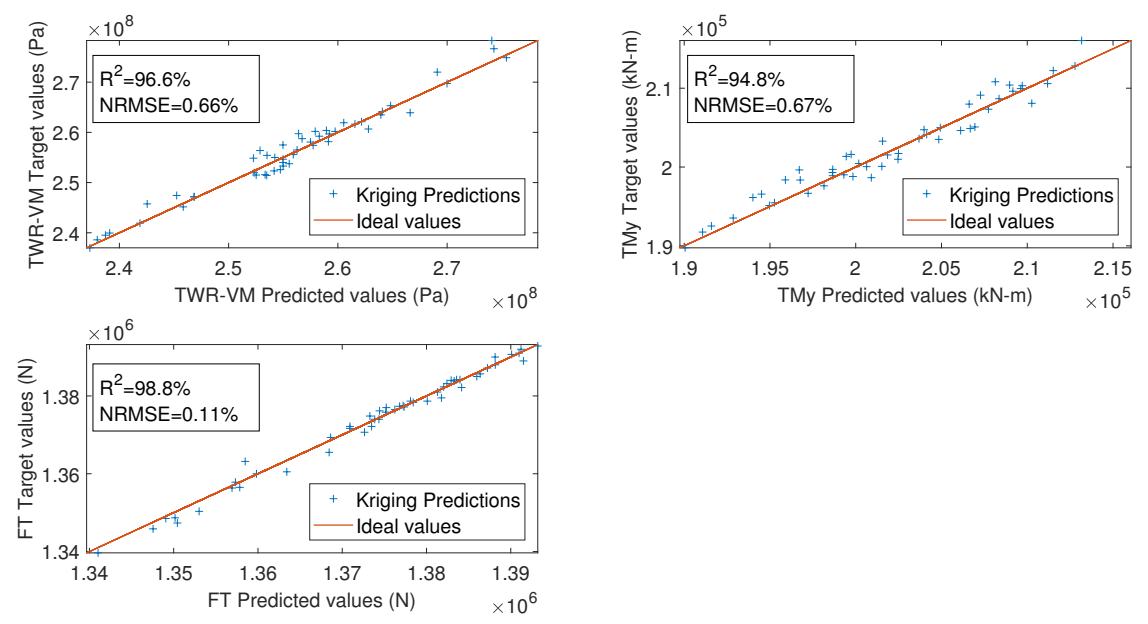

Fig. 18. DLC1.6a Kriging predictions Vs. Target values
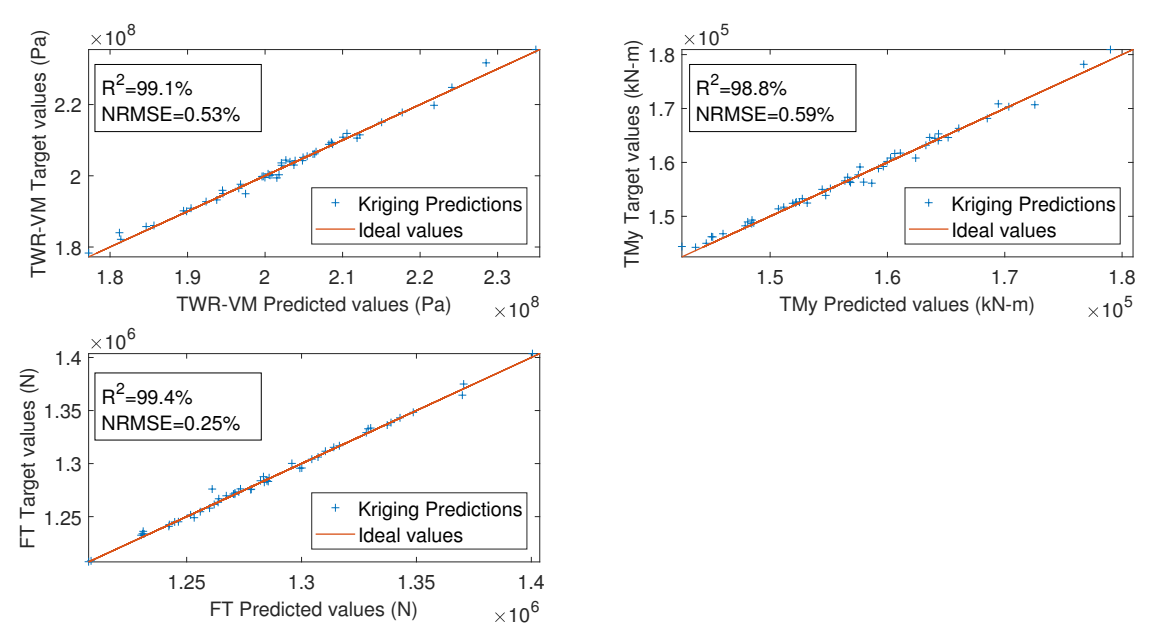

Fig. 19. DLC6.1a Kriging predictions Vs. Target values

574 where $L_{K}$ is the Kriging prediction, $b_{K}$ is the Kriging model bias estimated 575 using least squared method as given by Equation 14 and the error term $\epsilon_{t}$ 576 for each test sample point is determined using Equation 15.

$$
\begin{gathered}
b_{K}=\frac{\sum_{t=1}^{50}\left(L_{s i m} \cdot L_{K}\right)}{\sum_{t=1}^{50} L_{K}^{2}} \\
\epsilon_{t}=\frac{L_{\text {sim }(t)}}{b_{K} \cdot L_{K r i g}(t)}
\end{gathered}
$$


In Equation 15, $L_{\text {sim }}$ are the responses obtained using computationally expensive time-domain simulations and FE stress computation described in Section 4.1.1. The logarithm of the error $\epsilon_{t}$ and the mean error from the 50 sample points are used to estimate the standard deviation of the residuals $\sigma_{K}$, represented by Equation 16 and the $\mathrm{CoV}$ of the Kriging model $V_{K}$ is computed with Equation 17.

$$
\begin{gathered}
\sigma_{K}=\sqrt{\frac{1}{50-1} \sum_{t=1}^{50}\left(\ln \left(\epsilon_{t}\right)-\left[\frac{1}{50} \sum_{t=1}^{50} \ln \left(\epsilon_{t}\right)\right]\right)^{2}} \\
V_{K}=\sqrt{e^{\sigma_{K}^{2}}-1}
\end{gathered}
$$

The Kriging model bias and coefficient of variation for the load sensors investigated in this paper are presented in Table 5 .

Table 5. Kriging model uncertainty

\begin{tabular}{llllll}
\hline & & TWR-VM & TMy & FT & BCl \\
\hline \multirow{2}{*}{ DLC1.3 } & Bias & 1.001 & 1.001 & 1.000 & 1.000 \\
& CoV & 0.004 & 0.004 & 0.001 & 0.005 \\
\hline \multirow{2}{*}{ DLC1.6a } & Bias & 1.002 & 1.002 & 1.000 & 1.001 \\
& CoV & 0.006 & 0.007 & 0.001 & 0.011 \\
\hline \multirow{2}{*}{ DLC6.1a } & Bias & 1.001 & 1.002 & 1.001 & - \\
& CoV & 0.005 & 0.006 & 0.002 & - \\
\hline
\end{tabular}

The Kriging model uncertainties presented in Table 5 are subsequently incorporated in the formulation of limit state functions and reliability analysis presented in Section 6.2.

\section{Sensitivity analysis and reliability assessment}

\subsection{DLCs Sensitivity analysis}

To quantify the effect of the input random variables on the variance of the turbine responses under each DLC, global Sobol' indices [43] are computed. To evaluate the Sobol indices, Monte Carlo (MC)-based estimation 
is employed. Only a brief description is presented here, see Ref. [44] for copious details. First a matrix of size $N \times 2 \mathrm{~V}$ of random samples are generated from the distributions of the input variables, where $N$ is the number of MC samples, and $V$ is the number of input variables ( $V=4$ for DLC1.3 and $V=6$ for DLC1.6a and 6.1a). The $N \times 2 V$ matrix is then split equally into two matrices, $\boldsymbol{A}$ and $\boldsymbol{B}$ each having $N$ rows and $S$ columns. For each input variable $i ; i=1 \ldots V$, a third matrix $\boldsymbol{C}_{\boldsymbol{i}}$ is formed by taking all columns of $\boldsymbol{B}$ excluding the $i_{\text {th }}$ column which is taken from $\boldsymbol{A}$. Using the trained Kriging model, the responses are computed for all the input values in the matrices $\boldsymbol{A}, \boldsymbol{B}$, and $\boldsymbol{C}_{\boldsymbol{i}}$ as $N \times 1$ vectors $Y_{A}, Y_{B}$ and $Y_{C_{i}}$ respectively for each variable $i=1 \ldots V$. The total-effect Sobol index $\left(S_{T_{i}}\right)$ of each variable can be computed according to Equation 18.

$$
S_{T_{i}}=1-\frac{\frac{1}{N} \sum_{j=1}^{N}\left(Y_{B}^{(j)} Y_{C_{i}}^{(j)}\right)-\left(\frac{1}{N} \sum_{j=1}^{N} Y_{A}^{(j)}\right)^{2}}{\frac{1}{N} \sum_{j=1}^{N}\left(Y_{A}^{(j)}\right)^{2}-\left(\frac{1}{N} \sum_{j=1}^{N} Y_{A}^{(j)}\right)^{2}}
$$

An MC sample size, $N=10^{5}$ was used at a cost of $N(V+2)$, amounting to $6 \times 10^{5}$ evaluations. The total Sobol indices are reported in Fig. 20, 21 and 22 for DLC1.3, 1.6a, and 6.1a respectively. The stiffness of the

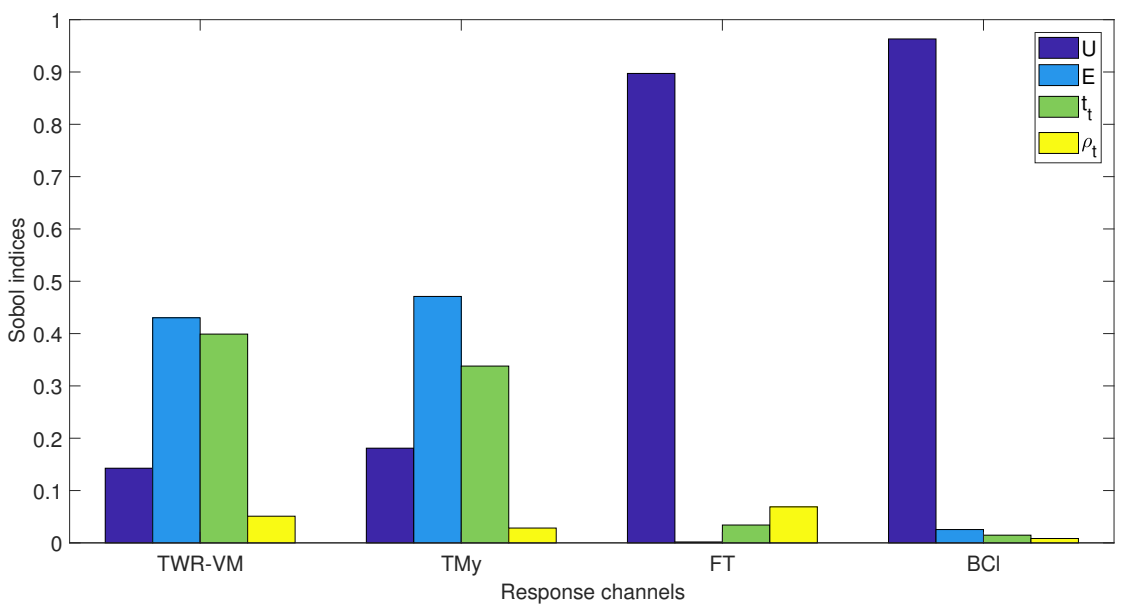

Fig. 20. DLC1.3 response sensitivity with respect to input random variables 


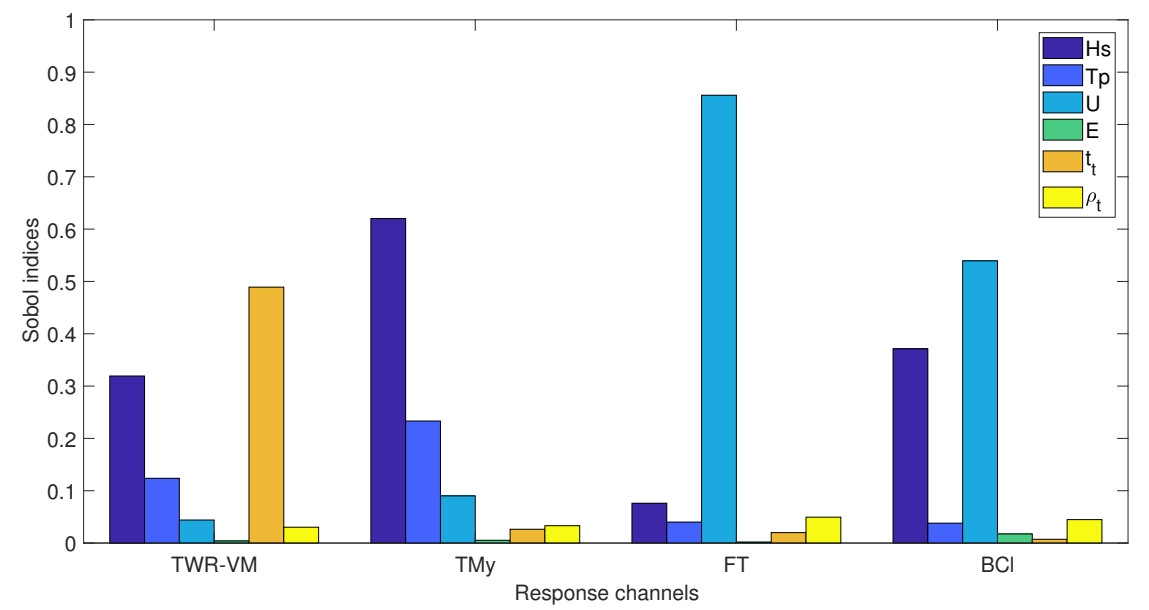

Fig. 21. DLC1.6a response sensitivity with respect to input random variables

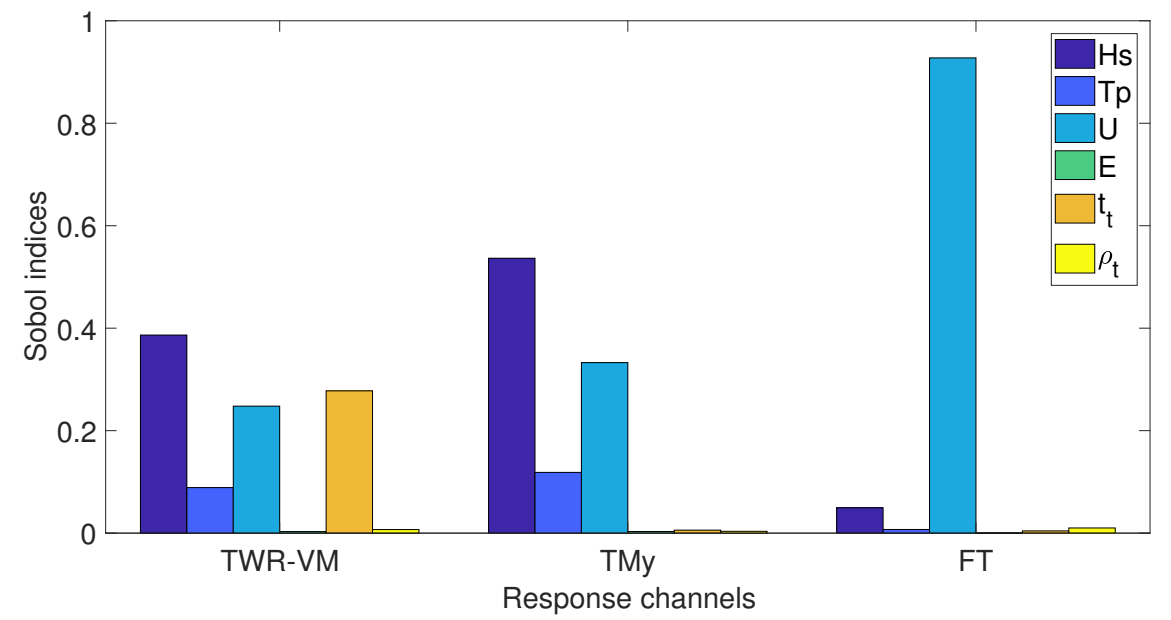

Fig. 22. DLC6.1a response sensitivity with respect to input random variables

tower characterized by $E$ and $t_{t}$ has the most effect on the von Mises stress $(T W R-V M)$ and moments $(T M y)$ in the tower for DLC1.3. The wind speed $U$ drives the fairlead tension $(F T)$ and blade-to-tower-clearance $(\mathrm{BCl})$ for all DLCs considered. When the sea state is not conditioned on wind speed as is the case with DLC1.6a and DLC6.1a, the wave height $H_{s}$ and the tower thickness $\left(t_{t}\right)$ had the most influence on the tower von Mises stress while $H_{s}$ and $U$ dominate the tower bending moment. These findings are very 
insightful in the design stages as the designer can readily tell which variables have most influence on key support structure load channels.

\subsection{Reliability Analysis}

Structural reliability is assessed by estimating the probability of failure of the structure. The demands or solicitation $(L)$ on the structure (i.e. load effects on the structure in the form of stresses, deflections, bending etc.) are compared to the capacity or resistance $(R)$ of the structure e.g. ultimate bending stress, yield strength, shear capacity etc. Generally, structural safety requires that the resistance of the structure be greater than the solicitation i.e. $R>L$, with $R \leq L$ implying failure of the structure. The failure probability is represented by Equation 19 .

$$
P_{f}=P[g(\boldsymbol{R}, \boldsymbol{L}) \leq 0]
$$

where $g(R, L)$ is the limit state function expressed in terms of the resistance random variable $R$ and the load random variable $L$. Simulation methods can be used to evaluate Equation 19 by sampling from the probability distributions of the input variables and evaluating the LSF for each sample point. The failure probability is then computed by Equation 20 .

$$
P_{f}=\frac{N_{f}}{N}
$$

where $N_{f}$ is the number of limit state violations (i.e. $\left.g(R, L) \leq 0\right)$ and $N$ is the total number of samples. A widely used sampling technique is the Monte Carlo Simulation (MCS) which involves direct sample-based estimation of the failure probability. A major drawback of MCS is the increased computational cost for the estimation of low failure probabilities. Subset Simulation (SS) offers computationally efficient alternative to MCS (see Ref. [45]). For sake of brevity, details of this approach is not provided in this work. Readers can refer to Ref. [46] for details of implementation within UQLab' reliability analysis toolbox. The total probability of failure due to a DLC, $P_{T}$ resulting from all the considered load cases in the DLC is computed according to Equation 21. 


$$
P_{T}=\sum_{L} P_{f}(L) f_{o c c}(L)
$$

where $P_{f}(L)$ is the failure probability computed for load case $L$ and $f_{\text {occ }}(L)$ is the probability of occurrence of the environmental conditions of load case $L$ (see Table 2 for DLC1.3 values of $f_{\text {occ }}(L)$ ). For DLC1.6a and DLC6.1a only the severest load case is used for reliability analysis. The 50-year metocean parameters are treated as uncertain parameters with a mean value and $\mathrm{CoV}$ to account for uncertainties associated with extrapolation techniques (quantifying this uncertainty was not within the scope of this paper, hence values for this uncertainty are based on engineering judgement). The failure probabilities under DLC1.6a and DLC6.1a conditions are therefore calculated based on the 50-year responses computed using realizations of the 50-year metocean parameters. It is worth mentioning that in general, 50-year responses calculated using extrapolated metocean parameters lead to different load levels compared to those obtained by extrapolating responses with proper account of the long term distribution of the environmental parameters [5]. However, the approach adopted here is considered to suffice within the scope of this paper.

\subsubsection{Verification of Kriging for reliability analysis}

The aim of this part of our study is to examine the efficacy of Kriging in the estimation of failure probability of FWT support structures with a look at the influence of the Kriging uncertainty. Given the computational cost of evaluating each LSF, the variance reducing alternative to the MCS, LHS is employed to enable sampling the tails of the distributions with limited sample size. A sample size of 1000 sample points requiring 6000 time-domain simulations is used. The failure probability is evaluated for three formulations of LSF defined describing the maximum von Mises stress observed in the tower exceeding the yield stress represented by Equation $22-24$.

$$
\begin{gathered}
g 1_{\text {case } 1}=F_{y}-\sigma_{\text {sim }} \\
g 1_{\text {case } 2}=F_{y}-b_{K r i g} \sigma_{K r i g} X_{K r i g}
\end{gathered}
$$




$$
g 1_{\text {case } 3}=F_{y}-\sigma_{K r i g}
$$

where $F_{y}$ is the yield stress of the tower. The yield stress is the resistance variable treated as a random variable with mean value set to $235 \mathrm{MPa}$ and $\mathrm{CoV}$ of 0.05 , modeled with a log-normal distribution. The structural demand is the maximum von Mises stress in the tower $\sigma_{\text {sim }}$ computed directly from aero-hydro-servo-elastic simulations and linear elastic stress computation and $\sigma_{\text {Krig }}$ computed using trained Kriging model. In Equation 23, $b_{\text {Krig }}$ is the Kriging model bias and $X_{\text {Krig }}$ represents realizations of a random variable with mean of 1 and the same $\mathrm{CoV}$ as the Kriging model. The 3 formulations of LSFs given by Equation 22 - 24 represents 3 cases for $P_{f}$ evaluations. Considering the huge cost of evaluating 1000 sample points, only several realizations of $F_{y}$ are generated and compared to the 1000 sample points of structural demand. For each case the input to the Kriging model remains unchanged. These are the 1000 sample points used in running the computationally expensive simulations. The mean $P_{f}$ computed for 1000:1000:10000 realizations of 1000 samples of $F_{y}$ are presented. The results are presented in Fig. 23.

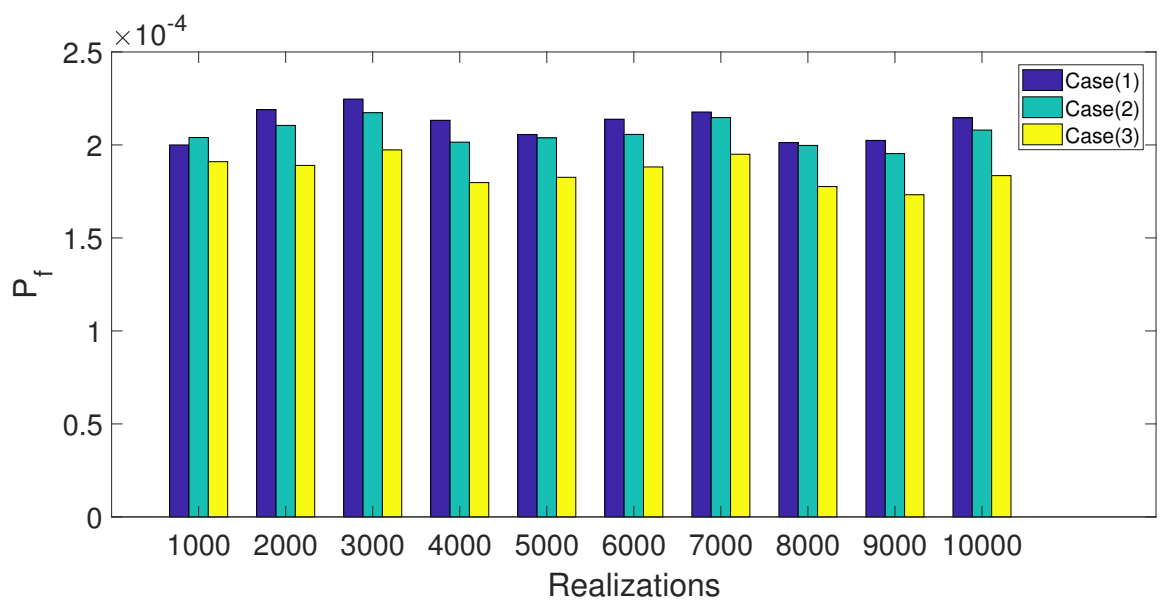

Fig. 23. Failure Probabilities for case 1, 2 and 3

The $P_{f}$ of case $(1)$ which is the ideal case converges to $\approx 2.1 \times 10^{-4}$. The results show that including the Kriging model bias and uncertainty in the LSF formulation as in case (2) resulted to a better match with case (1) as opposed 
to case (3) where these terms are not included. Incorporating the Kriging model bias and uncertainty into the LSF results in failure probabilities that are on average only $2.4 \%$ different from the true values as opposed to $12.1 \%$ when not incorporated. This shows that accurately quantifying and including the model uncertainties in the limit sate evaluation yields results close to reality.

\subsubsection{Estimation of failure probability for DLCs}

For each of the DLCs considered i.e. DLC1.3, 1.6a and 6.1a, the failure probability of the tower and mooring lines are evaluated using the trained Kriging models. The "true" mean wind speed, $U_{b i n}$ that produces maximum response of each load channel is first selected from Kriging response predictions of mean wind speed values $\left[\left(U_{b i n}^{*}-1\right): 0.1 \mathrm{~m} / \mathrm{s}:\left(U_{b i n}^{*}+1\right)\right]$, where $U_{b i n}^{*}$ is the mean wind speed bin suggested in Section 3.5 using a bin interval of $2 \mathrm{~m} / \mathrm{s}$. Some of the results are presented in Fig. 24 and 25. From Fig. 24 and 25, it is evident that the recommenced wind bin steps of $2 \mathrm{~m} / \mathrm{s}$ do not necessarily give sufficient resolution that captures the true extremes of turbine responses. The $13.6 \mathrm{~m} / \mathrm{s}$ wind bin produced higher loads compared to the $14 \mathrm{~m} / \mathrm{s}$ suggested in Section 3.5 , while the $11.5 \mathrm{~m} / \mathrm{s}$ wind bin which is very close to the rated wind speed of $11.4 \mathrm{~m} / \mathrm{s}$ produced the most extreme responses for DLC1.6a. To understand how the selection of wind bin affects structural failure computation, wind bins $U_{b i n}$ and $U_{b i n}^{*}$ are used in the reliability analysis that follows.
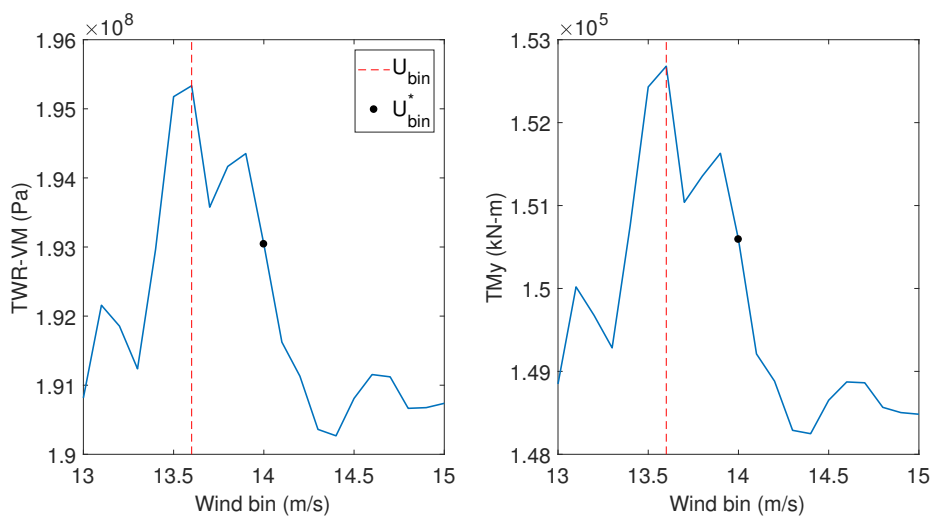

Fig. 24. DLC1.3 Wind speed bins

The LSFs considered for the tower are (1) G1: the tower von Mises stress exceeding yield limit described by Equation 25 and (2) G2: Simplified local 

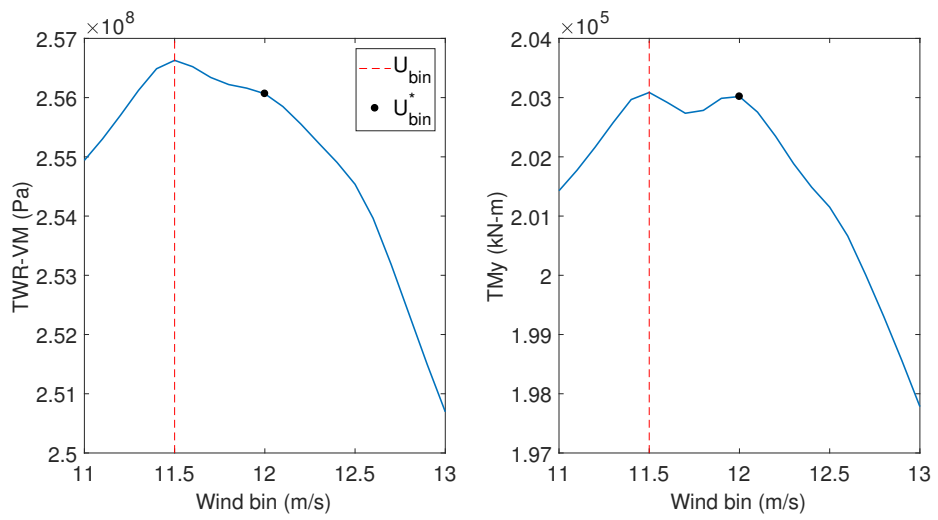

Fig. 25. DLC1.6a Wind speed bins.

buckling of the tower in similitude with that applied in the work by Sorenson et al. [16] (see Equation 26). The mooring line failure is governed by the LSF G3: the tension at the fairleads exceeding the minimum breaking strength of the mooring line given by Equation 27 .

$$
\begin{gathered}
G 1=F_{y} X_{y}-b_{\text {Krig }} \sigma_{\text {Krig }} X_{\text {Krig }} X_{\text {dyn }} X_{\text {str }} X_{\text {exp }} X_{\text {aero }} X_{\text {hydro }} \\
G 2=\frac{1}{6}\left(1-0.84 \frac{D}{t_{t}} \frac{X_{y} F_{y}}{E}\right)\left(D^{3}-\left(D-2 t_{t}\right)^{3}\right) X_{y} F_{y} \\
-b_{\text {Krig }} M_{\text {Krig }} X_{\text {Krig }} X_{\text {dyn }} X_{\text {str }} X_{\text {exp }} X_{\text {aero }} X_{\text {hydro }} \\
G 3=B_{L}-b_{\text {Krig }} T_{\text {Krig }} X_{\text {Krig }} X_{\text {dyn }} X_{\text {str }} X_{\text {exp }} X_{\text {aero } o} X_{\text {hydro }}
\end{gathered}
$$

The values of the variables in Equation 25-27 are given in Table 4. The $\boldsymbol{X}$ terms are stochastic variables which capture the uncertainties associated with the system. Their distributions and parameters are consistent with Ref. $[10,15,16,36]$. Uncertainty related to dynamic response modeling of the wind turbine which covers uncertainty in eigenfrequencies and damping ratios is modeled by $X_{d y n}, X_{s t r}$ models uncertainty related to the computation of load-effects, $X_{\exp }$ accounts for uncertainty associated with site assessment such as topography and terrain roughness. The use of quasi-steady BEM 
theory and assessment of aerodynamic drag and lift coefficients introduces uncertainty which is modeled by $X_{\text {aero }}$ while uncertainty related to the assessment of hydrodynamic drag and inertia coefficients is modeled by $X_{\text {hydro }}$. The Kriging model uncertainty is modeled by the random variable $X_{K r i g}$ with $b_{K r i g}$ as the Kriging model bias (computed in Section 5.5). Uncertainties in material and geometrical parameters also influence the design loads. These uncertainties are captured by the surrogate model and their influence quantified. In Equation 27, $T_{K r i g}$ is the maximum tension at the fairleads computed by the Kriging model. The breaking load of the mooring chain $B_{L}$ is derived from the chain nominal diameter $d=90 \mathrm{~mm}$ based on Equation 28 given in DNVGL-OS-E302 [47] for an R3 chain grade. Note that the mooring diameter of $90 \mathrm{~mm}$ is rather fictitious and has only been used here for sake of a generic analysis - more realistic values should be considered in order to relate the computed failure probabilities more rationally to the design life.

$$
B_{L}=0.0223 d^{2}(44-0.08 d)
$$

Using the reliability analysis toolbox UQLab [46], the probability of failures based on the limit state functions given by Equation 25 - 26 are computed using MCS of $10^{6}$ samples, while subset simulation is used to estimate the low failure probabilities for Equation 27 (readers can refer to Ref. [45] for details of this approach). For DLC1.3, failure probability is computed for three load cases $\left(L C_{1}, L C_{2}\right.$ and $\left.L C_{3}\right)$. Table 6 shows the calculated failure probabilities for the considered DLCs.

The failure probabilities for load case $L C_{1}, L C_{2}$ and $L C_{3}$ in Table 6 are reflective of the trend of the design driving loads plotted across wind bins with the most critical occurring with the $L C_{2}$ scenario. Considering all three load cases under DLC1.3, the total probability of failure due to DLC1.3 is less than $7 \times 10^{-4}$ for all LSFs. The mooring lines have $P_{f}$ values less than $10^{-16}$ for all DLCs. This is because the mooring system is designed to always have a catenary shape with a layed-down part before the anchorage which effectively limits the tension in the lines. Results in Table 6 for DLC1.3 and DLC6.1a show levels that are compatible with target probability of failure values of $2 \times 10^{-4}-10^{-3}$ used in the calibration of partial safety factors in the 
Table 6. Probability of failure due to load cases.

\begin{tabular}{lllll}
\hline \multicolumn{2}{c}{ DLC } & \multicolumn{3}{c}{ LSF } \\
\cline { 2 - 5 } & & G1 & G2 & G3 \\
\hline \multirow{2}{*}{1.3} & $L C_{1}$ & $4.2 \mathrm{E}-05\left(2.9 \mathrm{E}-05^{*}\right)$ & $4.3 \mathrm{E}-05\left(2.6 \mathrm{E}-05^{*}\right)$ & $<10^{-16}$ \\
& $L C_{2}$ & $9.56 \mathrm{E}-04\left(6.73 \mathrm{E}-04^{*}\right)$ & $1.48 \mathrm{E}-03\left(9.35 \mathrm{E}-04^{*}\right)$ & $<10^{-16}$ \\
& $L C_{3}$ & $1.31 \mathrm{E}-04\left(1.01 \mathrm{E}-04^{*}\right)$ & $1.54 \mathrm{E}-04\left(9.7 \mathrm{E}-05^{*}\right)$ & $<10^{-16}$ \\
& $L C_{T}$ & $4.476 \mathrm{E}-04\left(3.16 \mathrm{E}-04^{*}\right)$ & $6.76 \mathrm{E}-04\left(4.26 \mathrm{E}-04^{*}\right)$ & $<10^{-16}$ \\
\hline $1.6 \mathrm{a}$ & & $4.61 \mathrm{E}-02\left(4.43 \mathrm{E}-02^{*}\right)$ & $8.08 \mathrm{E}-02\left(7.25 \mathrm{E}-02^{*}\right)$ & $<10^{-16}$ \\
\hline $6.1 \mathrm{a}$ & \multicolumn{2}{c}{$2.2 \mathrm{E}-03\left(1.94 \mathrm{E}-03^{* *}\right)$} & $3.74 \mathrm{E}-03\left(2.75 \mathrm{E}-03^{* *}\right)$ & $<10^{-16}$ \\
\hline$*: U_{\text {bin }}^{*}$ is used, material and geometric uncertainties neglected \\
$* *:$ material and geometric uncertainties neglected, $U_{\text {bin }}^{*}$ not applicable \\
$L C_{T}$ : Combination of $L C_{1}, L C_{2}$ and $L C_{3}$
\end{tabular}

IEC 61400-1 [4] and IEC 61400-3 [5] design standards (see Ref. [16, 36]). It is noted that the probabilities of failure for DLC1.6a are slightly away from the target values range. This is due to high loads produced by excitation from the 50-year sea state combined with action of controllers and rotor dynamics. Similar high loads are reported in Ref. [19, 20] as well. Improving the hydrodynamic damping of the system is one of the solutions proposed by Jonkman and Matha [20]. With such improvement, the probability of failure for DLC1.6a is expected to fall within the target value range.

Neglecting the influence of material and geometric uncertainties on the controlling loads is usually common in studies on wind turbine reliability analysis. So also the use of $2 \mathrm{~m} / \mathrm{s}$ wind bin interval. Together, these can amount to as much as $39 \%$ reduction in failure probability (e.g. $L C_{2}$ ). Although not included in the results presented, using $0.1 \mathrm{~m} / \mathrm{s}$ bin interval gave wind bin values that amounted to $\approx 19 \%$ and $18 \%$ increase in the total failure probability for LSF G1 and G2 respectively. The inclusion of all the considered modeling uncertainties amounted to failure probabilities that are about $10^{12}$ times higher than those computed without taking modeling uncertainties into account. This is attributable to structural demands in terms of load effect been multiplied by factors as high as 2.3 (from the product of realizations of the $\boldsymbol{X}$ random variables), implying greater number of limit state violations. Evidently, modeling uncertainties significantly increase failure probability of structural members and should not be neglected in the design process. 


\section{Conclusions}

A framework for robust reliability analysis of FWT support structures under ULS design for IEC 61400-3 [5] DLC1.3, 1.6a and 6.1a was presented. The first part of this work established design driving metocean conditions for the considered load cases and a ranking of DLCs based on selected response channels was presented. The power production DLC1.6a resulted in the most critical loads. This is attributable to the combined action of rotor dynamics, control system loads and severe sea state. Subsequently load effect computation in terms of structural stress evaluation was presented. The methodology adopted is hinged on linear elastic FEA. This linearization enabled the conversion of time series of lumped loads into stress time series - amounting to huge computational savings when time-domain simulations are imperative provided the structural loads are not expected to lead to non-linear deformation.

After training the Kriging models, a validation of Kriging for estimating structural failure probability was presented. Using 6000 simulations, making up 1000 sample points, it was shown that correctly estimating and incorporating the Kriging model bias and uncertainty into the LSF results in failure probabilities that are on average only $2.4 \%$ different from the true values as opposed to $12.1 \%$ when not incorporated.

Finally, failure of the tower under yield and local buckling limit states is evaluated as well as failure of the mooring lines. By training the Kriging models around the most severe wind bin determined from load analysis using $2 \mathrm{~m} / \mathrm{s}$ bin interval, a more accurate design driving wind bin is determined using $0.1 \mathrm{~m} / \mathrm{s}$ bin interval. This resulted to between $19 \%-18 \%$ increase in computed failure probabilities for DLC1.3. When material and geometric uncertainties are accounted for, together with selecting the "true" design driving wind bin, failure probability is reduced by up to $39 \%$ of values obtained when these uncertainties are neglected. The findings of this study show the influence of various uncertainties in the design of wind turbine support structures and the presented methodology for capturing these uncertainties would be highly beneficial when incorporated in reliability-based optimization schemes and partial safety factor calibration.

\section{Acknowledgments}

The first author would like to thank the Petroleum Technology Development Fund (PTDF), Nigeria for the funding of this PhD research. 


\section{References}

[1] C. Zou, Q. Zhao, G. Zhang, B. Xiong, Energy revolution: From a fossil energy era to a new energy era, Natural Gas Industry B 3 (2016) 1-11.

[2] S. Sánchez, J.-S. López-Gutiérrez, V. Negro, M. D. Esteban, Foundations in offshore wind farms: Evolution, characteristics and range of use. analysis of main dimensional parameters in monopile foundations, Journal of Marine Science and Engineering 7 (2019) 441.

[3] G. Katsouris, A. Marina, Cost Modelling of Floating Wind Farms, ECN, 2016.

[4] IEC, Iec 61400-1: Wind turbines part 1: Design requirements, International Electrotechnical Commission (2005).

[5] IEC, Iec 61400-3: Wind turbines-part 1: Design requirements, International Electrotechnical Commission, Geneva 64 (2005).

[6] Z. Jiang, W. Hu, W. Dong, Z. Gao, Z. Ren, Structural reliability analysis of wind turbines: A review, Energies 10 (2017) 2099.

[7] A. Morató, S. Sriramula, N. Krishnan, Kriging models for aero-elastic simulations and reliability analysis of offshore wind turbine support structures, Ships and Offshore Structures 14 (2019) 545-558.

[8] R. M. Slot, J. D. Sørensen, B. Sudret, L. Svenningsen, M. L. Thøgersen, Surrogate model uncertainty in wind turbine reliability assessment, Renewable Energy (2019).

[9] L. E. S. Stieng, M. Muskulus, Reliability-based design optimization of offshore wind turbine support structures using analytical sensitivities and factorized uncertainty modeling (2020).

[10] H. Yang, Y. Zhu, Q. Lu, J. Zhang, Dynamic reliability based design optimization of the tripod sub-structure of offshore wind turbines, Renewable Energy 78 (2015) 16-25.

[11] M. Muskulus, S. Schafhirt, Reliability-based design of wind turbine support structures, in: Proceedings of the Symposium on Reliability of Engineering System, Hangzhou, China, volume 1517, 2015. 
[12] B. Standard, Eurocode - basis of structural design, 2002.

[13] L. Haid, G. Stewart, J. Jonkman, A. Robertson, M. Lackner, D. Matha, Simulation-length requirements in the loads analysis of offshore floating wind turbines, in: International Conference on Offshore Mechanics and Arctic Engineering, volume 55423, American Society of Mechanical Engineers, 2013, p. V008T09A091.

[14] L. B. D. GL, Qualification of innovative floating substructures for 10mw wind turbines and water depths greater than 50m (2015).

[15] W. N. Wandji, A. Natarajan, N. Dimitrov, Development and design of a semi-floater substructure for multi-megawatt wind turbines at $50+\mathrm{m}$ water depths, Ocean Engineering 125 (2016) 226-237.

[16] J. D. Sørensen, H. S. Toft, Probabilistic design of wind turbines, Energies 3 (2010) 241-257.

[17] J. Jonkman, S. Butterfield, W. Musial, G. Scott, Definition of a 5-MW reference wind turbine for offshore system development, Technical Report, National Renewable Energy Lab.(NREL), Golden, CO (United States), 2009.

[18] J. Jonkman, Definition of the Floating System for Phase IV of OC3, Technical Report, National Renewable Energy Lab.(NREL), Golden, CO (United States), 2010.

[19] A. N. Robertson, J. M. Jonkman, et al., Loads analysis of several offshore floating wind turbine concepts, in: The Twenty-first International Offshore and Polar Engineering Conference, International Society of Offshore and Polar Engineers, 2011.

[20] J. Jonkman, D. Matha, Dynamics of offshore floating wind turbines - analysis of three concepts, Wind Energy 14 (2011) 557-569.

[21] B. Jonkman, J. Jonkman, Fast v8. 16.00 a-bjj, National Renewable Energy Laboratory (2016).

[22] A. C. Young, A. J. Goupee, H. J. Dagher, A. M. Viselli, Methodology for optimizing composite towers for use on floating wind turbines, Journal of Renewable and Sustainable Energy 9 (2017) 033305. 
[23] M. Muskulus, Pareto-optimal evaluation of ultimate limit states in offshore wind turbine structural analysis, Energies 8 (2015) 14026-14039.

[24] K. Wei, S. R. Arwade, A. T. Myers, Incremental wind-wave analysis of the structural capacity of offshore wind turbine support structures under extreme loading, Engineering Structures 79 (2014) 58-69.

[25] D. H. Kim, S. G. Lee, Reliability analysis of offshore wind turbine support structures under extreme ocean environmental loads, Renewable energy 79 (2015) 161-166.

[26] Y. Hsu, W.-F. Wu, Y.-C. Chang, Reliability analysis of wind turbine towers, Procedia Engineering 79 (2014) 218-224.

[27] J. Velarde, C. Kramhøft, J. D. Sørensen, Global sensitivity analysis of offshore wind turbine foundation fatigue loads, Renewable Energy 140 (2019) 177 - 189. URL: http://www.sciencedirect.com/science/article/pii/S096014811930360X. doi:https://doi.org/10.1016/j.renene.2019.03.055.

[28] K. Johannessen, T. S. Meling, S. Hayer, et al., Joint distribution for wind and waves in the northern north sea, in: The Eleventh International Offshore and Polar Engineering Conference, International Society of Offshore and Polar Engineers, 2001.

[29] J. M. Jonkman, Dynamics modeling and loads analysis of an offshore floating wind turbine, Technical Report, National Renewable Energy Lab.(NREL), Golden, CO (United States), 2007.

[30] J. Jonkman, M. Buhl, New developments for the nwtc's fast aeroelastic hawt simulator, in: 42nd AIAA Aerospace Sciences Meeting and Exhibit, 2004, p. 504.

[31] J. M. Jonkman, Modeling of the UAE wind turbine for refinement of FAST $\{-\}$ AD, Technical Report, National Renewable Energy Lab., Golden, CO (US), 2003.

[32] B. J. Jonkman, M. L. Buhl Jr, TurbSim user's guide, Technical Report, National Renewable Energy Lab.(NREL), Golden, CO (United States), 2006. 
[33] T. R. Kane, D. A. Levinson, Dynamics, theory and applications, McGraw Hill, 1985.

[34] E. Bossanyi, Gh bladed version 3.6 user manual, Garrad Hassan and Partners Limited Document 2 (2003).

[35] M. D. Shields, J. Zhang, The generalization of latin hypercube sampling, Reliability Engineering \& System Safety 148 (2016) 96-108.

[36] N. J. Tarp-Johansen, Partial safety factors and characteristic values for combined extreme wind and wave load effects, J. Sol. Energy Eng. 127 (2005) 242-252.

[37] G. Matheron, Principles of geostatistics, Economic geology 58 (1963) $1246-1266$.

[38] J. Sacks, W. J. Welch, T. J. Mitchell, H. P. Wynn, Design and analysis of computer experiments, Statistical science (1989) 409-423.

[39] I. Kaymaz, Application of kriging method to structural reliability problems, Structural Safety 27 (2005) 133-151.

[40] C. Lataniotis, S. Marelli, B. Sudret, Uqlab user manual-kriging (gaussian process modelling), Report UQLab-V0 (2015) 9-105.

[41] F. A. C. Viana, G. Venter, V. Balabanov, V. Steffen Jr, On how to implement an affordable optimal latin hypercube, in: Proceedings of the 19th Congress of Mechanical Engineering (COBEM'07), 2007.

[42] G. Zarraonandia, C. Bittencourt, Reliability-based calibration of partial safety factors for the horizontal axis tidal turbine standard for certification, in: Proceedings of the ASME 33rd International Conference on Ocean, Offshore and Arctic Engineering (OMAE 2014), San Francisco, CA, USA, 2014, pp. 8-13.

[43] I. M. Sobol, Sensitivity estimates for nonlinear mathematical models, Mathematical modelling and computational experiments 1 (1993) 407414 .

[44] A. Saltelli, M. Ratto, T. Andres, F. Campolongo, J. Cariboni, D. Gatelli, M. Saisana, S. Tarantola, Global sensitivity analysis: the primer, John Wiley \& Sons, 2008. 
939

940

941

942

943

944

945

946

[45] S.-K. Au, J. L. Beck, Estimation of small failure probabilities in high dimensions by subset simulation, Probabilistic engineering mechanics 16 (2001) 263-277.

[46] S. Marelli, R. Schöbi, B. Sudret, Uqlab user manual-structural reliability (rare events estimation), Structural Reliability, Report UQLab-V0 (2016) 9-107.

[47] DNVGL, Offshore standard dnvgl-os-e302: Offshore mooring chain, 2015. 